\title{
Public acceptance and perception of autonomous vehicles: a comprehensive review
}

\author{
Kareem Othman ${ }^{1,2}$ (D) \\ Received: 8 October 2020 / Accepted: 28 January 2021 / Published online: 26 February 2021 \\ (c) The Author(s), under exclusive licence to Springer Nature Switzerland AG part of Springer Nature 2021
}

\begin{abstract}
Autonomous vehicles (AVs) or self-driving cars have the potential to provide many benefits such as improving mobility and reducing the energy and emissions consumed, travel time, and vehicle ownership. Thus, in the last few years, both research and industry have put significant efforts to develop AVs. However, laws and regulations are not ready yet for this switch and the legal sector is unable to take the lead but follow the development of AVs. Besides, the social acceptance is considered as a main key factor for the success of any new technology. Despite the enthusiastic speculation of AVs, little is known about the public acceptance and perception of the AVs technology or the factors that influence the public acceptance. This paper reviews the previous studies that focuses on testing the public acceptance and perception of AVs and sketches out the main trends in this area to provide some directions and recommendations for the future. This paper focuses on the influence of safety, ethics, liability, regulations, and the recent pandemic on the public acceptance of AVs.
\end{abstract}

Keywords Autonomous vehicles $\cdot$ Safety $\cdot$ Accidents $\cdot$ Ethics $\cdot$ Liability and regulations $\cdot$ Implications on public acceptance

\section{Introduction}

Research in vehicle automation has launched many years ago. In the 1920s, research focused on the vehicle-to-vehicle communication system using radio waves [1]. This was followed by the invention of the electromagnetic guidance system in the 1940s [2]. In the 1980s, the first autonomous vehicle (AV) system was invented when Mercedes-Benz partnered with Bundeswehr University in Munich [3]. The invention of the first autonomous vehicle (AV) or self-driving car was a milestone that attracted many manufacturers and since this point many companies have been competing for introducing AVs [4]. According to the National Highway and Transportation Safety Administration, there are 5 levels of AVs' functionality. Level 0: no automation and the driver perform all the driving tasks. Level 1: driver assistance system which means that the driver takes control of the vehicle while some driving assistance features are added

Kareem Othman

kareem.othman@mail.utoronto.ca;

karemmohamed1993@cu.edu.eg

1 Civil Engineering Department, University of Toronto, $35 \mathrm{St}$ George St, Toronto, ON M5S 1A4, Canada

2 Civil Engineering Department, Cairo University, Giza, Egypt to the vehicle; level 2: partial automation which means that the vehicle has an automated function, but the driver must be engaged in the driving tasks; level 3: conditional automation which means that drivers must always be ready to take control of the vehicle; level 4: high automation which means that the vehicle can drive autonomously but the driver might have the option to take control; and level 5: full automation which means that the vehicle can take control of the vehicle under all conditions [5].

In the last decade, autonomous vehicles (AVs) have witnessed tremendous improvement because of the significant effort dedicated to AVs from both research and industry [6]. In 2009, Google launched a project with the vision of introducing AVs by 2020 [7]. Uber and Volvo introduced the third generation of their AVs and it was expected that they would test it by 2020 [8]. In 2014, Apple launched its AV project "Titan" and they announced that their AVs will be available by 2016 but the project faced many issues and it is expected that their AVs will be available between 2023 and 2025 [9]. Moreover, new startup companies launched with the vision of providing AVs. For example, Zoox was launched in 2014 and its value was increasing rapidly and reached $3.2 \$$ billion by 2018 [10]. From cities perspective, many cities allowed AVs testing. Now, in the US, 20 states allowed 
the deployment and testing of AV s on their public roads $[11,12]$. The main point of these testing or pilot studies is to understand the implications of AVs and the attitude of the public towards AVs.

Over the past few years, the implications of AVs have been discussed intensively. While AVs are expected to provide a large number of benefits, they are also associated with risks or drawbacks. It is expected that AVs will increase the level of comfort [13], improve productivity $[14,15]$, and improve mobility for people with limited transportation [16]. On the other hand, it is expected that AVs might increase the vehicle kilometers traveled, and the level of congestion because of the extra empty trips [17]. The advantages and disadvantages of autonomous technology are coexisting, and AV technology is growing rapidly as both research and industry have put significant efforts to develop AVs. While AVs are often discussed in regard to their implications, benefits, technological development, and technological challenges, less attention has been paid to the public acceptance and perception of AVs. The process of innovation diffusion may not always be smooth. For many new technologies, the non-technological issues are more likely to be a barrier toward the adoption of this technology than the technological ones, and for sure AVs are one of these technologies. IEEE reported to CNN that the "biggest barrier to pervasive adoption of driverless cars may have nothing to do with technology but will be general public acceptance. While the average driver may grasp the basic benefits of autonomous cars-increased fuel efficiency and safety, along with a reduction in traffic-it may not be enough to get them to let go of the steering wheel" [18]. Thus, the main objective of this paper is to understand and analyze the main factors that influence the public acceptance of $\mathrm{AVs}$ as follows:

- AVs safety and the influence of the perceived level of safety on the public attitude towards AVs.

- The ethical dilemma and challenges facing the programming of the vehicle.

- Liability and regulations and their implications of the public acceptance of AVs.

- The implications of the current pandemic on the perception and acceptance of AVs.

- Reviewing the small but growing body of work examining public attitudes to AVs, which has tended to focus on a range of predictor variables including demographic characteristics, specific psychographic attributes, and willingness to pay additional amounts for AV technology. Previous work has primarily involved quantitative surveys.

- Then, the paper reveals some gaps in the literature and provides some recommendations for the future.

\section{AVs' safety}

Safety is a major pillar for the success of any new technology. While most of the previous studies assume that AVs can enhance traffic safety by more than $90 \%[19,20]$ due to the elimination of the human error, safety studies show that other factors might replace the human error and increase the rate of collision. Additionally, safety studies show that AVs' safety is extremely complex and still in the early stages.

In 2015, Sivak and Schoettle found that there are many factors which contribute to accidents and the contribution of these factors will change with the introduction of AVs. For example, while some vehicle failure might become obsolete, some other failures will show up. Lighting failure might become obsolete as vehicles will not depend on the visual input, but this might be a problem during the transition period when both conventional and autonomous vehicles share the same roads, as human drivers might not be able to see the vehicles ahead. Additionally, the complexity of the system and sensors will increase the vehicular failure rates which might increase the rate of accidents. Moreover, sometimes accidents are unavoidable. In a situation, when a person suddenly appears in front of an AV and the distance ahead is not enough to brake, the AV will not have a choice but crashing. Although AVs react faster than the human drivers as they eliminate the perception and reaction time, the braking distance might not be enough to stop [21]. Aside from the vehicular failure, some other factors contribute to accidents such as the other road users. For example, at the intersection level, pedestrians were responsible for $80 \%$ of accidents [22]. Finally, the behavior of $\mathrm{AVs}$ is unpredictable in many conditions and might be affected by the environmental and weather conditions of the surrounding area.

In 2017, two studies discussed the safety of AVs. Koopman and Wagner showed that safety of AVs is a complex process that requires coordination between different disciplines starting from machine learning, to sensor development, to testing AVs, and developing a standard testing process [23]. Klara focused on the main issue or challenges for realizing AVs' safety. First, there is no standard for testing AVs. For example, the use of the driving test will not be feasible for AVs, because this test will make sure that AVs have the ability to perform the basic driving tasks, while the behavior of AVs in different or complex conditions will not be tested [24]. May be one good approach is the one followed by Tesla. In 2016, Tesla announced that their vehicles have the ability to drive autonomously but this feature would operate in the shadow mode in which the vehicle make decisions, but these decisions are not executed, instead the human driver actions 
are the actions executed [24]. The main benefit of this approach is to gather information about AVs' decisions and compare these decisions with the human driver actions and train the vehicles to mimic the human drivers in the future. Also, another benefit of this approach is to test AVs and make sure whether they take the right decisions or not and how AVs learn and improve over time. Second, there is no agreement on the definition of the safety of AVs. While some people think that $\mathrm{AVs}$ must be allowed once they provide a higher safety level than human driving, others will not trust machines and will not accept the idea of putting their lives in the hands of machines [25].

There are many examples of AVs' failures (next section discuss AVs accidents in details), but one of the most famous AV failures occurred in 2018 in the US when one of Uber (Volvo) AVs detected a passenger crossing the road and the vehicle chose not to make an action and continued driving until it hit the pedestrian. The National Transportation Safety Board (NTSB) reported that the vehicle detected the pedestrian six seconds before the accident but did not brake until $1.3 \mathrm{~s}$ before hitting the fatal accident [26]. Later in 2018, the CEO of Waymo declared that he does not believe that AVs will be able to drive autonomously and in many conditions, it will require some human interaction [27, 28].

Research on the safety of AVs started at the University of Illinois more than two decades ago. In 2020, they reported that safety of AVs is a sophisticated process because of the complexity of the system that is equipped with more than 50 processors and required to run million lines of codes to make instantaneous decisions. Additionally, the variety of conditions and situations make it hard or almost impossible to train $\mathrm{AVs}$ in all these conditions. When the team analyzed AVs' data in 2020, results showed that AVs are much more likely to be involved in accidents than human drivers. This study defined the AV accident event as the event when the technology fails to appropriately handle the situations, disengage the technology, and ask the human driver to take over. This situation is considered a system failure and might lead to an accident. Thus, it is considered as an accident [29].

In the next section, details on AVs' reported accidents (as examples of vehicular failure) all over the world and the impact of these accidents or news on public acceptance will be discussed.

\section{Implications of AVs' safety on the social acceptance}

\subsection{Public perception of AVs' safety and trust in AVs}

Social acceptance is the main key for the success of any new technology. Accidents of AVs might have an influence on the public acceptance, especially it was found that some people cannot trust machines [30]. This subsection shed light on the public perception of the safety of AVs and the level of trust in AVs. One survey study by Casley et al. was conducted in the US with 467 respondents and with the aim of understanding the impact of three factors on the public acceptance of AVs. These three factors are the safety of the system, the cost of the technology, and the liability issue [31]. The following bullets are the main questions asked in the survey and their results:

- Respondents were asked to rank the importance of safety, costs, and laws on their perception of AVs. 82\% of the respondents ranked safety as the most important aspect in order to adopt $\mathrm{AVs}, 12 \%$ believe that laws are the most important aspect, and $6 \%$ choose costs. These results show that safety is a top priority for people and illustrate that people will not adopt AVs until they make sure that this new technology is safe. Additionally, laws are ranked as the second factor that influences the public acceptance towards AVs.

- Respondents were asked if they trust an autonomous system and if they believe that the autonomous system can drive better than a normal human driver. $74 \%$ of the respondents do not trust AVs nor believe AVs can perform better than a normal driver, while $26 \%$ of the respondents do.

- Respondents were asked if they have concerns regarding the AV system. Respondents were very concerned about the safety aspects of the AV system that only $6.9 \%$ of the respondents have no concerns regarding the $\mathrm{AV}$ system. On the other hand, $74 \%$ of the respondents believe that AVs would be prone to malfunction, 57\% of the respondents were concerned about the system's poor awareness of its surrounding environment, 52\% were concerned about programming issues, and 50\% were concerned about the poor control of the system such as braking and steering.

- Respondents were asked "How many years after the technology is introduced to the market would you feel comfortable purchasing a car with an autonomous driving system?" 4.4 responded they will consider purchasing AVs immediately, 7.7\% would feel comfortable using AVs after a year, $25.9 \%$ would feel comfortable using AVs in 1-2 years, $31.6 \%$ would feel comfortable in 3-4 years, and $30.5 \%$ would feel comfortable in more than 4 years. These results can translate the trust issue as most people (62.5\%) prefer to wait more than two years before considering AVs. Probably, the rationality behind this is that people do not trust new technologies at early stages especially if these technologies will affect their own safety and lives. Generally, any new technology is not totally perfect and include some 
faults. Thus, people prefer to wait until the maturation of this technology before adopting it.

Another survey in Austin, US shed light on the trust issue in AVs' technology. During this survey, respondents were asked to choose the reasons that discourage them from adopting AVs. $41 \%$ of the respondents stated that they will not consider AVs because of the lack of trust in the technology, $24 \%$ stated they will not consider AVs because of their concerns about the safety of this new system, and $22 \%$ will not consider AVs because of the high costs. Results of this survey show that both safety and lack of trust in AVs are the main obstacles that prevent people from adopting AVs [32].

On a third international survey by Rezaei, and Caulfield, people were not likely to believe in the safety and security of AVs' operation. Results show that $44 \%$ of the respondents do not believe that AVs are safer than a normal human driver, while $25 \%$ believe AVs are safer. Additionally, $66 \%$ of respondents responded that they will not feel safe if the vehicle does not have a steering wheel, while only $14 \%$ have no problem if the vehicle does not have a steering wheel, which in turn indicates that a large proportion of people do not trust the system. Finally, respondents were asked whether they are willing to adapt AVs once they are available or wait to see the opinion of the early adopters. Results show that only $13 \%$ will adapt AVs once they are in the market, and $87 \%$ of the respondents will wait to see the opinion of the early adaptors [33].

Different other surveys all over the world and across the years show that people have high levels of concerns regarding the safety of AVs as follows:

- The survey by Schoettle and Sivak in US, UK and Australia shows that $92 \%$ of the respondents are highly concerned about the safety of the AV in poor weather and about the interaction between the vehicle and pedestrians [34].

- The survey by Schoettle and Sivak in the US shows that $69 \%$ of the respondents are highly concerned about the safety of the AV system [35].

- The survey by Kyriakidis et al. that received response from 109 countries shows that $76 \%$ of the respondents are highly concerned about the safety of the AV system [36].

- The survey by Greaves et al. in Australia shows that $68 \%$ of the respondents are highly concerned about the safety of the AV system [37].

Thus, the safety of AVs is paramount. If the vehicles are not safe, they are significantly less desirable, regardless of their benefits. The perceived safety, or rather the perceived lack of safety of AVs, is what will truly sway the opinions of potential buyers. Given this, AVs' manufacturers should emphasize the safety of AVs and prove to the public that operating an autonomous car is not a risky endeavor.

While it is expected that the public acceptance would increase with time, results of Šinko et al. showed that this is not the case when they compared their survey results with Schoettle and Sivak results. Results show that people become more negative about AVs in 2017 with an average acceptance of 3.3 out of 5 than in 2014 with an average acceptance of 3.6-4.3 out of 5 in different countries [38]. One reason might be the news about the first fatal accident that involves an AV which accompanied Šinko et al. study. Thus, it is essential to investigate the influence of accidents on the public perception of AVs.

\subsection{Implications of AVs' accidents on the public attitude and trust in AVs}

\subsubsection{Exploring the impact of a single accident (event) on the public attitude towards AVs}

Studies on the implications of AVs' accidents on the public attitude are very rare. To the best of the author's knowledge, only one study on the literature focuses on the implications of AVs' accidents on the public attitude. After one AV accident in 2019, Jefferson and McDonald quantified the implications of this accident on the public attitude using semantic analysis for social media data, specifically Twitter data. The main goal of this study is to understand the change in the public perception of AVs before and after the accident. Specifically, this study investigated the change in conversations about autonomous vehicles three days before the event, on the day the event was first reported, and three days after the event [39]. The main advantage of the semantic analysis for social media data is that, unlike traditional surveys, it provides a large amount of information. For example, more than $45 \%$ of adults aged 18-24 years and $30 \%$ of adults aged $25-49$ years use Twitter in the US. Additionally, Twitter holds over 300 million active accounts worldwide [40]. The analysis shows that the word "crash" does not appear before the accident, but it becomes a common word after the accident. Additionally, the common words three days before the accident were "Amazon", "Startup", and "invest". However, on the day the crash was first reported, the most frequent terms were "vehicle", "autopilot", and "drive". Three days after the crash was reported, the most frequent terms were "vehicle", "crash", and "autopilot". Figure 1 shows the most frequent words used on Twitter for the three cases. Additionally, during the semantic analysis process text is analyzed to measure its positivity or negativity. The analysis consists of looking up words or phrases in a sentiment dictionary and calculating a sentiment score. Sentiment dictionaries map words and phrases to emotional scores, 
Fig. 1 FREQUENTLY words tweeted prior to the crash, on the day the incident was first reported, and 3 days after the crash [39]
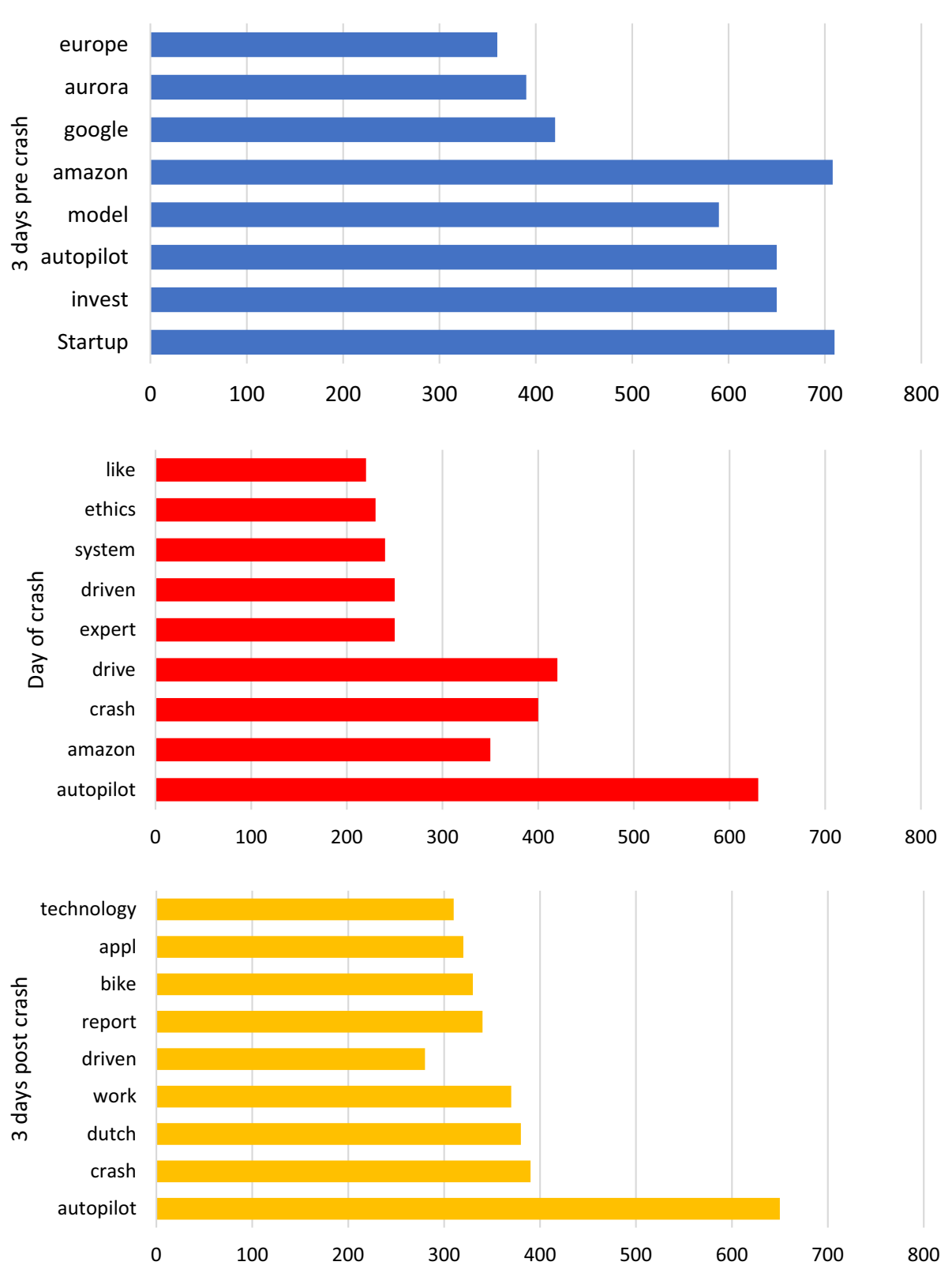

with higher emotions leading to larger scores, and other words leading to zero scores. Figure 2 shows the results of the semantic analysis. The figure illustrates that across time, both positive and negative sentiment decreased following the crash. However, the drop in the positive sentiment was much larger than the decrease in negative sentiment [39]. These results indicate that a large proportion of the positive tweets disappeared (almost half), while the negative tweets almost remain the same as before the accident, which means that a large number of people who were positive towards AVs before the accident might became worried about the technology. Additionally, this accident reduced the level of trust in the technology especially for people with positive attitude that they stopped tweeting about the positives of AVs.

\subsubsection{Exploring the impact of AVs' accidents on the public attitude}

The previous study shows how the news about one accident was discussed intensively and it became a trend on the social media platform. Such discussion regarding the safety of AVs has a significant impact on the public concerns and trust in this technology. Actually, news regarding AVs' accidents are always covered extensively that Tesla's CEO Elon Musk always complaint about the extensive media coverage of their AVs' accidents. He stated that "One of the things I should mention, that frankly has been quite disturbing to me is the degree of media coverage of Autopilot crashes, which are basically almost non-relative to the paucity of media coverage of the 1.2 million people that die every year in manual crashes. It 


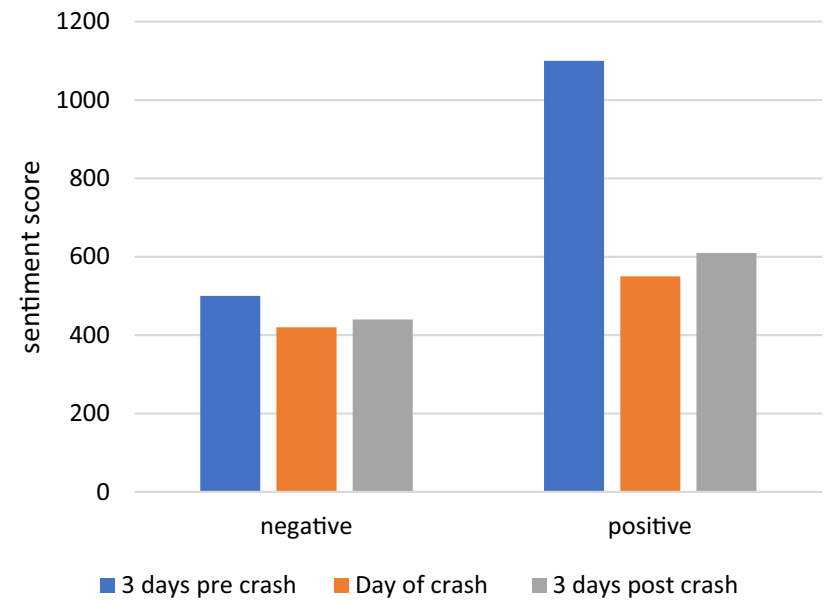

Fig. 2 Semantic score for the three cases [39]

is something that I think does not reflect well upon the media. It really does not." [41].

The previous study by Jefferson and McDonald investigated the impact of one single accident on the public perception of AVs. Thus, it is critical to understand the influence of AVs' accidents on the public attitude over time. Thus, Table 1 and Fig. 3 summarize AVs' accidents and show the relation between the level of fear of AVs [42-44] and the number of accidents reported. Table 1 summarizes AVs' accidents, provide details about every accident, and finally show a very small group of newspapers (with the largest number of subscribers) that covered these accidents on the same day to show how extensive the media covered the news about every accident. This extensive coverage means that this news will be delivered to every person in the world and increase the level of fear of this technology. Figure 3 shows that both the public fear and the number of reported accidents have the same trend. While the number of AVs' accidents increases, people become more scared of AVs, and while the number of AVs' accidents decline the percentage of people afraid of AVs also declines. Thus, the safety of AVs is paramount. If the vehicles are not safe, they are significantly less desirable, regardless of their benefits. The perceived safety, or rather the perceived lack of safety of $\mathrm{AVs}$, is what will truly sway the opinions of potential buyers. Given this, AVs' manufacturers should emphasize the safety of AVs and prove to the public that operating an autonomous car is not a risky endeavor.

\section{Synopsis on the technological realization of AVs (sensor fusion, computer vision, and fault tolerance)}

At this point, it is also important to discuss the technological development to support AVs' safety. While AVs' vision and object detection are hard tasks, AVs' developers are currently adapting a new system that depends on diversity (different types of sensors) and redundancy (overlapping between sensors to detect objects with high accuracy) to support AVs' visibility and decision-making. Commonly, three major sensors are used: camera, radar, and lidar. These three sensors can provide AVs with the required information of the surrounding information to operate safely. While cameras are the best way for visualization, they have their own limitations. Camera sensors cannot calculate the distance to objects accurately and in many cases camera sensors find it difficult to detect objects in many conditions such as fog, rain, nighttime, or snow. Radars can support cameras in low visibility conditions to detect objects. Additionally, radars can detect speeds and distances accurately. In fact, most of the conventional cars used nowadays are supported with cameras and radar sensors to support specific tasks such as driver and parking assistance. However, the information provided by camera and radar sensors is not sufficient for a vehicle to operate autonomously. As a result, lidar sensors can provide a good solution. Lidar is a sensor that measures distances by pulsing lasers. Lidar sensors allow AVs to construct 3D views. Thus, AVs' developers are using the three types of sensors to enhance the visibility of AVs through a process that is called sensor fusion. During the sensor fusion process, sensors inputs are fed into a powerful and centralized computer to combine the data and support the vehicle decision-making process. Thus, sensor fusion support AVs to fuse various information from the sensor suite, instead of relying on a single and unreliable sensor. Also, sensor fusion provides redundancy as the same areas are covered by different types of sensors to support the vehicle decision [103-107]. While detecting information is an important task, AVs must interpret the environment to make decisions. Manufacturers are employing artificial intelligence and machine learning to support AVs' decisions. However, the use of machine learning and artificial intelligence is a very complex process that requires massive data to train the vehicle to make the right decision [104].

AVs' navigation is a difficult task because of a variety of obstacles such as the weather conditions [108]. These obstacles create uncertainty when the vehicle is unable to gain sufficient information from the surrounding environment, which makes it difficult for the vehicle to precisely follow a pre-defined path [109]. This section focuses on 


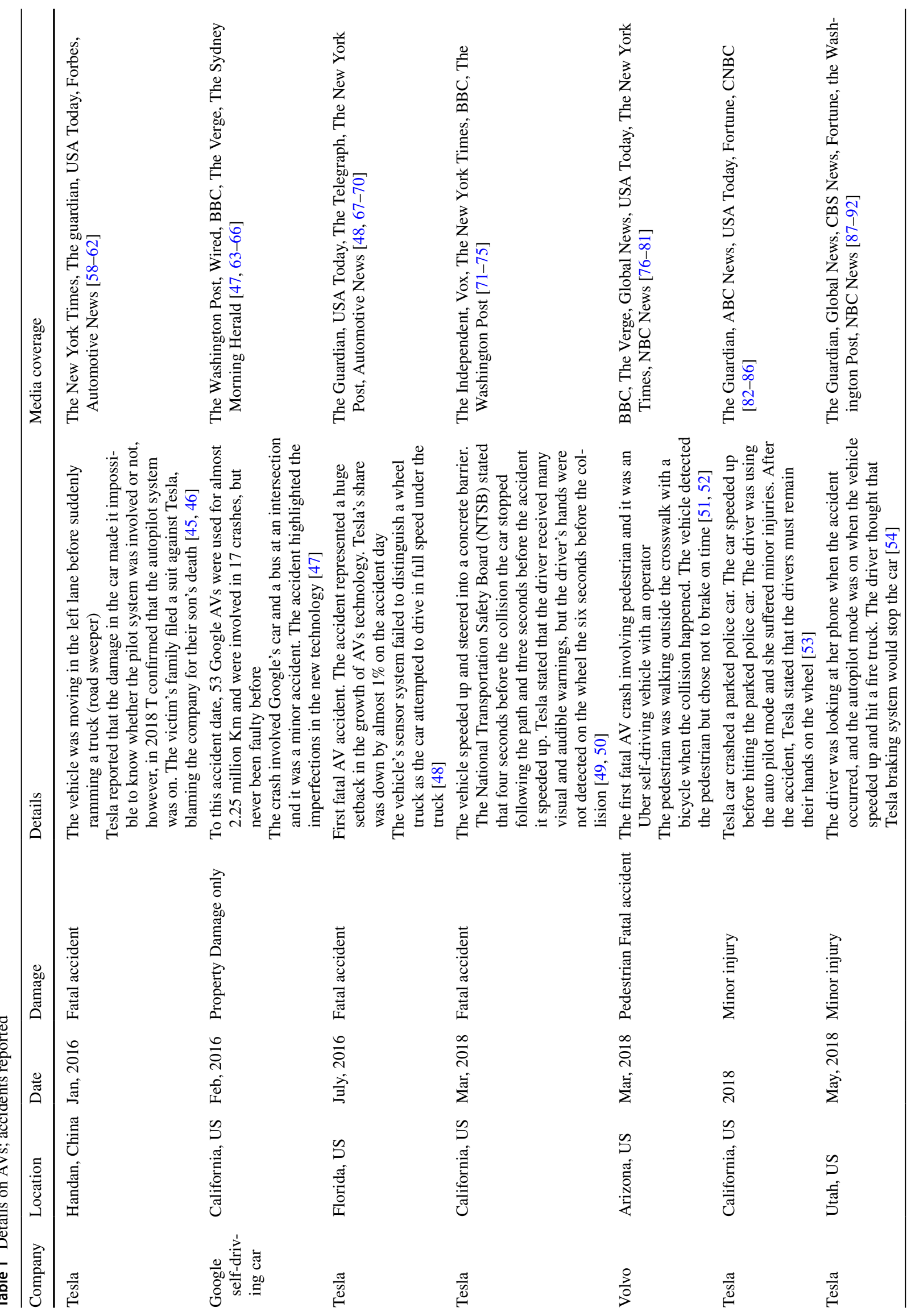




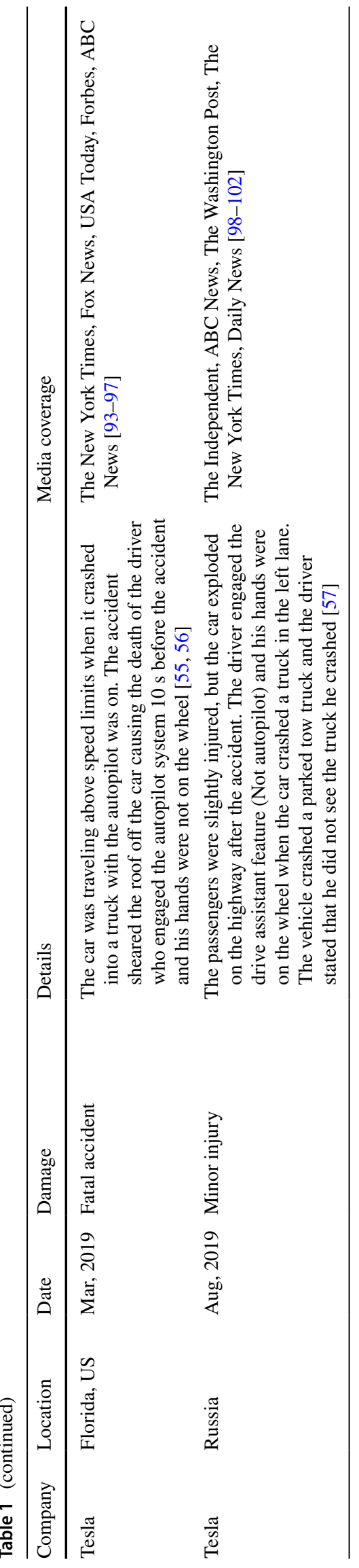

three major fields: sensor fusion, computer vision, and fault tolerance. Generally, the process of integrating data and knowledge from serval sensors to enhance the accuracy is called data fusion. The main goal of the data fusion process is to reduce the probability of errors and improve the reliability of the system. Due to the increase in the number of sensors used in AVs and the data fusion process, the probability of software or hardware failures increases in terms of sensor failures, actuators malfunctions, and processing failures [110]. Thus, a fault tolerance strategy is critical to improve the performance of the vehicle. Fault tolerance aims at preventing small faults from developing into serious failures.

\subsection{Sensor fusion}

Data fusion can be defined as the process of combining data from different sensors in order to enhance the overall performance and accuracy of the vehicle, this accuracy cannot be achieved relying on a single sensor. This section summarizes and shed light on sensor fusion methods and results from recent studies. Generally, state estimation methods are based on the control theory that employs the laws of probability to compute a vector state from a vector measurement or a stream of vector measurements. In general, there are three estimation methods as follows:

- Machine learning methods

- Kalman filter methods

- Particle filter methods

\subsubsection{Machine learning (ML) methods}

ML is an estimation method that is based on the probabilistic theory. Generally, probabilistic methods are convenient when the state variable follows an unknown probability distribution [111]. The main drawback of this method is that it needs analytical or empirical model of a particular sensor to compute the likelihood function and ascertain prior distribution values. Additionally, this method might underestimate the distribution variance, which might lead to data biases. However, this problem can be avoided by increasing the number of data points $(N)$ used as the variance of the sample equals the variance of the original data at the limit $N \rightarrow \infty$. Over the past few years, many research studies employed the particle filter approach for sensor fusion such as $[112,113]$.

\subsubsection{The Kalman filter methods}

The Kalman filter is the most common estimation technique. This method is mainly used to fuse low-level data. In case the system can be represented as a linear model and the error can be represented as a Gaussian noise, the 
Fig. 3 Change in the number of accidents and people fear over years

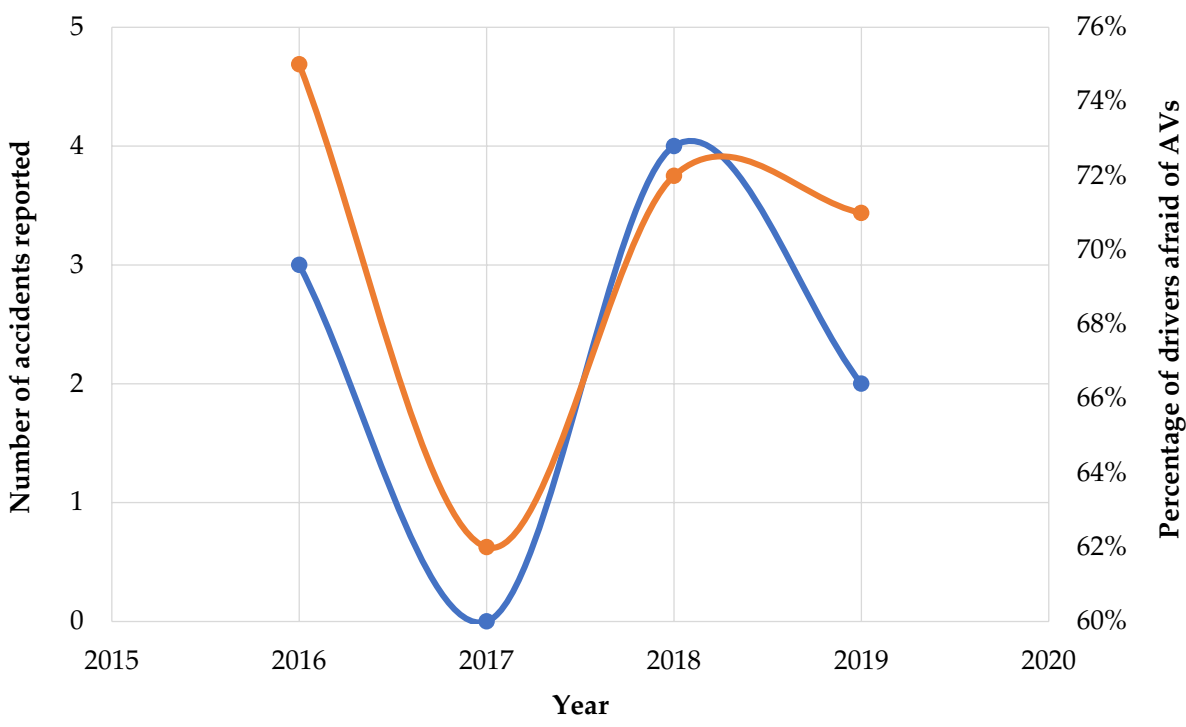

- Number of Reported Accident $\quad-$ Percentage of Drivers Afraid of Avs filters can estimate the optimal statistical estimations [114]. However, for the nonlinear dynamic models, other methods are used such as the modified Kalman filter [115]. The modified Kalman filter is the most popular method used for data fusion in robotics. However, the major drawback of this approach is the computation of the Jacobians which is extremely expensive, but some research has been made on this area to reduce the computational costs such as applying linearization, but this approach may introduce new errors in the filter and thus makes it more unstable [109]. Over the past few years, an extensive number of studies employed the Kalman filter approach in order to fuse data from different sources such as:

- In 2006, Caron et al. employed the Kalman filter approach based on fuzzy subsets to fuse multi-sensor GPS and inertial measurement unit (IMU) data. The simulation was carried out using GPS and IMU data from real vehicle tests. In many cases, the GPS signal was not credible because of the drifts on the INS. In these cases, the Kalman filter was fed by the IMU data [116].

- In $2015, \mathrm{Li}$ et al. proposed a hybrid system for reliable navigation of vehicles. This system employees the Kalman filter to ascertain the filtering fusion. The proposed system fuses data from low-cost sensors such as GPS and acts to enhance the performance over the integration scheme of these sensors [117].

- Xian et al. (2015) introduced a system that integrates data from stereo camera and a low-cost GPS to obtain the exact motion estimation for the AV navigation. An algorithm was developed to estimate the motion within the overall system based on an iterative extended Kalman filter (IEKF). This system benefits from the inertial sensor's fast response and the visual sensor's slow drift [118]. Figure 4 illustrates the integration flowchart between the GPS or IMU data.

- In 2016, Ryu et al. integrated the GPS with INS using the extended Kalman filter and the unscented Kalman filter
Fig. 4 The integration flowchart between the GPS or IMU data. The flowchart is divided into four parts with different colors, namely inertial navigation part (black part in the flowchart); image processing (blue) consisting of feature detecting, tracking and outlier rejection; the part of iterative extended Kalman filter (green) and the system states management part (red) [118]. (Color figure online)

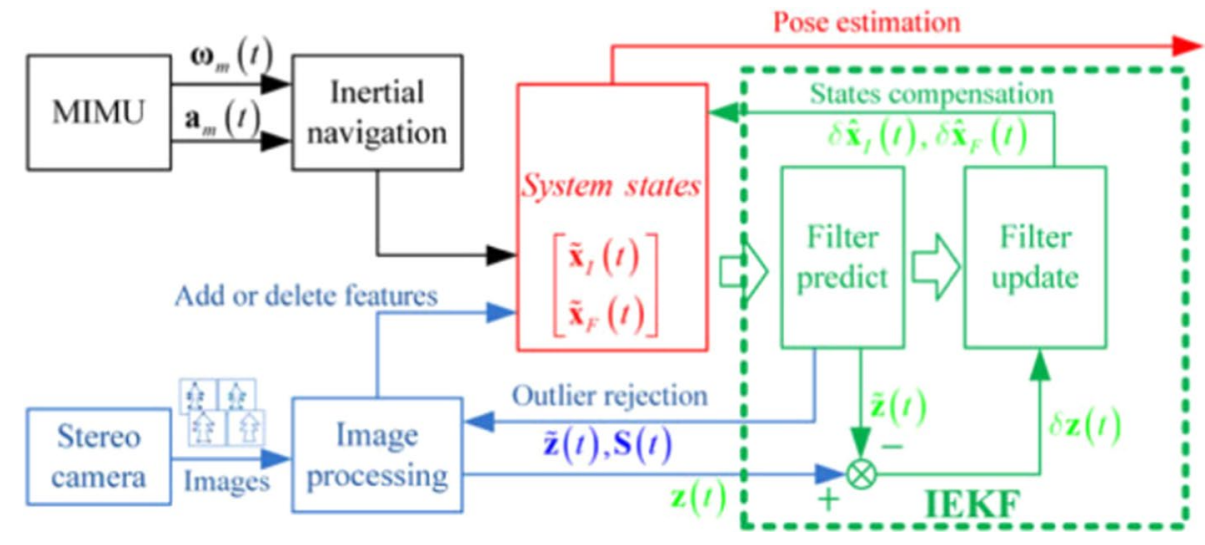


in order to reduce the errors associated with low cots sensors and estimate positions accurately. Moreover, this method was assessed in a field test and results show that the obtained data are accurate [119].

\subsubsection{Particle filter methods}

This approach is based on the iterative implementation of Monte Carlo technique [120] in order to solve the filtering problem. This approach builds upon the density function using several random samples called particles. During the filtering process, the particles that receive the same state variables are employed and weights are added to each particle according to the quality of the particle. The final estimation is a weighted sum of all the particles. Generally, there are two phases for each algorithm: the prediction phase, and the updating phase. During the prediction phase, particles are modified based on an existing model, taking into account the impact of the random noise in order to simulate the noise effect. During the updating phase, the weights assigned to each particle are re-evaluated based on the observations from the last available sensor. Over the past few years, many research studies employed the particle filter approach for sensor fusion such as [121-124].

\subsection{Computer vision}

The image processing algorithms required for an AV to operate safely are extremely complex. Additionally, AVs should be equipped with the required power to process images onboard so that the vehicle can react to the surrounding world ad avoid objects. Additionally, another challenge facing AVs is the required hardware platform to facilitate the image processing task because this platform should be lightweight, small, and provide the required processing power with low consumption rate in order to operate autonomously, for long times, and without having to be re-charged. Over the past few years, an extensive number of studies focused on the computer vision or image recognition process of AVs such as:

- In 2010, Oniga and Nedevschi proposed the digital elevation map (DEM) based approach which is one of the major contributions to the obstacle detection research field. This approach builds the DEM and another two density maps from a set of 3-D points in order to represent the adjacent surrounding environment. Additionally, an obstacle detection algorithm was developed to identify the density of these 3-D points per DEM. Then, the algorithm classifies each cell in the DEM as an obstacle or road object based on a slope-based threshold criterion. Figure 5 shows an example output of the proposed system [125]. in 2014, Danescu and Nedevschi built upon the DEM approach and applied the particle filter strategy in performing DEM tracking, which followed the dynamic DEM approach [126].

- In 2011, Min et al. developed an image recognition algorithm for AVs based support vector machine to identify objects received from a camera. This algorithm was able to measure the headway distance between the vehicle itself and the vehicle in front. Additionally, this algorithm was able to recognize lanes and avoid the vehicle dropping out of the lane by measuring the distance between the wheels and the lane. Moreover, this algorithm was able to recognize signs through pattern matching and color matching tasks so that the vehicle was able to react appropriately [127].

- In 2016, Schaub et al. developed an obstacle avoidance system that relies solely on the data received from a monocular camera stream. The developed algorithm was able to identify dynamic obstacles using two-stage clustering and an optical flow. Due to the direct cou-
Fig. 5 Example output of the algorithm provided by Oniga and Nedevschi-road area with blue, obstacles with red and traffic isles with yellow [125]. (Color figure online)

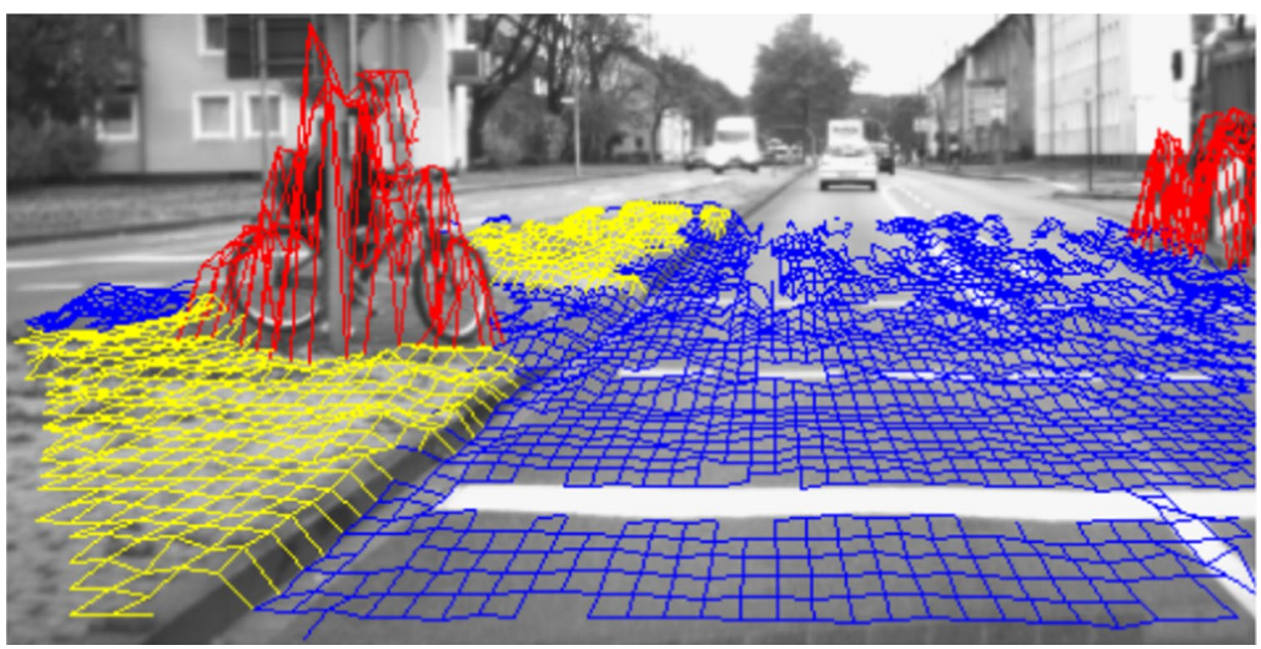


pling between the controls of the vehicle and the image motions, without a transformation into the Cartesian space, it was possible to explicitly consider and evade dynamic obstacles utilizing image data from a monocular camera [128].

\subsection{Fault tolerance}

\subsubsection{Introduction to fault tolerance}

There are two sources of fault that might occur in AVs as follows: hardware defect, or software imperfections. In order of appearance, faults can be divided into two categories hard or soft faults. Hard faults occur suddenly when the data changes from the normal state to a faulty state. Soft faults occur gradually. The period when the data are delivered in a faulty state is called the service outage period [129]. There are four main approaches to avoid service outage periods as follows:

- Fault prevention: this approach focuses on preventing errors.

- Fault removal: this approach focuses on the application of corrective actions [130].

- Fault forecasting: this approach focuses on estimating the number of faults and their consequences. this approach is usually used for faults that cannot be removed.

- Fault tolerance: this approach denotes the ability of the system to avoid failure with the presence of faults.

While fault preventions and fault removal focus on the avoidance or removal of faults, fault forecasting and fault tolerance deal with focus on avoiding the system failure with the existence of faults to avoid unplanned behaviors [131]. In complex systems, it is not possible to achieve a fault-free system, so the fault forecasting or fault tolerance approaches are the preferred approaches. For AVs, the fault tolerance approach is the most common approach followed [129]. The most common methods used for sensor fault detection are the analytical methods or the model-based models that require mathematical modeling of the system, inputs, and outputs in order to produce the detected features. Theses feature, then, are compared with the variables of the system to estimate the residual values. The residual is compared with the nominal values to detect faults and their locations. Mathematical models are very popular for fault tolerant control systems because their parameters can usually be obtained. However, in complex tasks, it is hard to develop a good and representative mathematical model, and this might not be possible. In those complex cases, other techniques such as artificial neural networks are used for fault detection and diagnosis [132]. In the last few years, researchers focused on implementing fault tolerant modules for AVs in many areas such as the vehicle navigation sensors. Generally, the architectures developed for the safe navigation of AVs can be divided into two categories centralized architectures and federated architectures.

Centralized architectures employ one global filter to process the measurements of all the local sensors. This architecture can detect hard sensor failures through the detection of residuals. However, the single centralized filter architecture is not powerful enough to detect soft sensor failures. On the other hand, federated architectures consist of a group of filters that work in parallel and a master filter. This architecture isolates the data from faulty sensors before integrating it into the system as the local filters fuse the data independently from a specific sensor with the common reference sensor information. Then, the information is fused in the master filter to generate the best estimations [133, 134]. After identifying the error, two methods are used for the fault recovery: direct redundancy or analytical redundancy. In the direct redundancy, an additional module is employed to replace the faulty one. However, the analytical redundancy implies to utilize the working modules to complete the tasks which failed [135].

\subsubsection{Fault tolerance systems for AVs}

The main objective of the FTC is to detect faults in the system and allow the system to perform conveniently even with the presence of some faults. Generally, FTC can be classified into two categories: passive and active control. Active FTC depends on fault detection and diagnosis process to monitor the system performance, while passive FTC deals with predefined faults by using a specially designed fixed controller. The main advantage of the active FTC is the ability to detect and identify faults in the system and reconfigure the controller online and in real-time [136-138]. Any FTC system should have the ability to deal with many challenges such as the fast response time to avoid the degradation of the system performance to an undesired level, and failure coverage to allow the system to deal with as many failures as possible. Over the past few years, fault tolerance systems attracted many researchers such as:

- Fourlas et al. focused on developing a model-based fault diagnosis, for a four-wheel skid steering mobile robot. The main objective of this study is to develop a FTC system that detects faults as early as possible and recalculate command inputs in order to achieve fault tolerance, which offered a feasible solution to the residual generation of nonlinear systems. The proposed system consists of two parts. First, the fault detection module accepts, as inputs, the measurement of the linear and angular velocity of the SSMR robot and further decides the appropriate type of fault to use according to the detection algo- 
rithm. Second, the fault accommodation module, which accepts, as inputs, the type of fault required as well as the measurement of the linear and angular velocity [139].

- Vlantis et al. study dealt with faults for an omni-directional mobile platform using four mechanized wheels moving within a flat and constrained workspace along with several static-based obstacles. conventional and dipolar Navigation Functions were combined using adaptive control techniques to deal with the parametric uncertainty associated with the robot and its dynamics [140].

- Bader et al. study developed an FTC system that is based on the traditional duplication comparison. The developed approach offers detecting and diagnosing faults in a data fusion mechanism. The fault tolerance process consisted of two main tasks: error detection and system recovery. In the error detection tasks, the system detects the erroneous state of the system before errors are propagated. Then, the system develops an error-free state to be substituted in place of an erroneous state. Figure 6 shows the architecture of the FTC system developed in this study [141].

Finally, the process of AVs' navigation is a complex process that requires coordination between different disciplines starting from machine learning, to sensor development, to testing AVs, and developing a standard testing process. As shown above, the public acceptance of AVs is significantly influenced by the perceived level of safety of AVs. If the vehicles are not safe, they are significantly less desirable, regardless of their benefits. The perceived safety, or rather the perceived lack of safety of AVs, is what will truly sway the opinions of potential buyers. Thus, the technological maturation is a critical key factor for the acceptance and success of AVs.

\section{Ethical dilemma and implications on the public behavior and acceptance}

\subsection{The ethical dilemma and social challenge}

Similar to AVs' accidents, ethics have a significant influence on the public acceptance of any new technology. AVs' ethical dilemma is similar to the famous trolley paradox; however, the paradox will be more frequent for the case of AVs. This dilemma can occur when a collision is unavoidable, so the vehicle's software has to choose how and what to crash. [142-145]. To understand this dilemma, imagine a situation where an AV has no chance to avoid collision and must choose between swerving right to hit a young girl or swerving left to hit an old woman or moving ahead and hit both. While it seems better to swerve to the right direction and hit the young girl to reduce the vehicle loss as the vehicle might choose to hit the lighter object to protect the owner, it also looks better to swerve left and hit the old person as the young girl still has her entire life. However, based on the code of ethics the two choices are unethical as the Institute of Electrical and Electronics Engineers (IEEE) emphasizes on treating fairly all persons regardless of their race, religion, gender, disability, age, national origin, sexual orientation, gender identity, or gender expression" [146].

One solution might be providing a cost function that can be used to minimize the collision impacts or risks. This cost function will be able to transfer ethics into cost according to their weights then optimize the function to provide the ideal result. However, this approach will not be appropriable for complex situations as the vehicle will have to evaluate the different types of damage (injury, death, property damage) to the parts involved in the accident then reduce the overall costs [147, 148], but such a solution requires a significant amount of information about the surrounding objects and results of different actions. Unfortunately, this approach might not be convenient at all. Let us imagine a situation where a vehicle cannot avoid
Fig. 6 Duplication-comparison architecture for fault tolerance in multisensory perception [141]

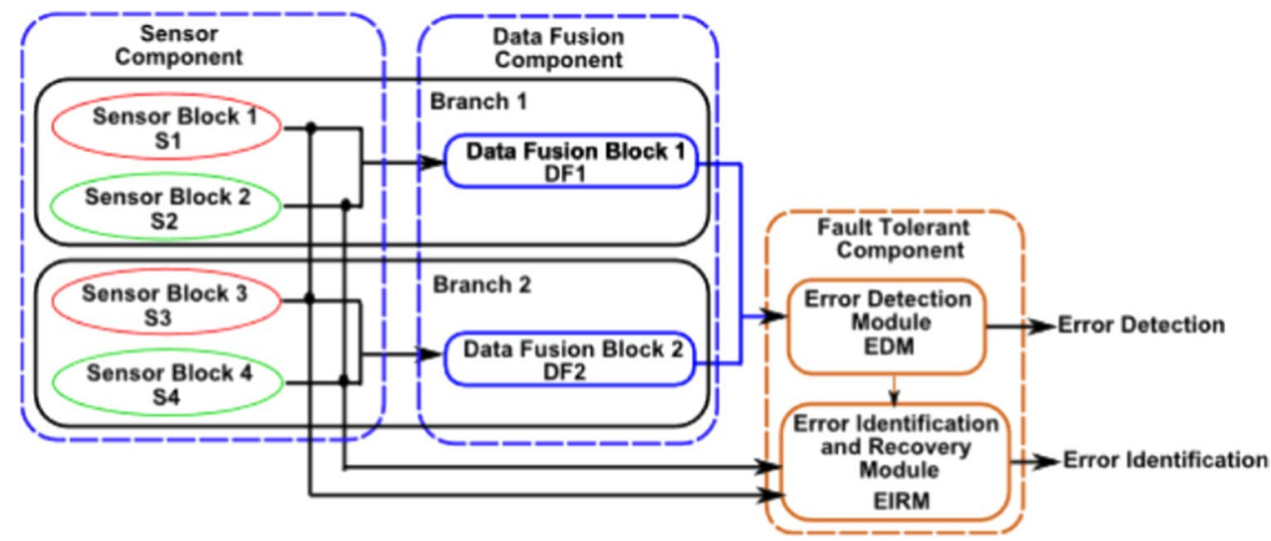


a crash and has to choose one of two motorcycles to hit one wearing a helmet and the other do not. Based on the cost function, the vehicle will take the action that minimizes the cost function which is hitting the one with the helmet as his chances of survival are higher. However, this behavior will discourage people from wearing helmets to avoid being crashed [147].

Another suggestion is to allow vehicle owners to determine the vehicle's ethical values, as people will not use a vehicle that might sacrifice themselves in a life-or-death dilemma. On the other hand, it might look better that laws should intervene and determine the ethical standards that all AVs should follow [142, 149]. In 2016, the German government appointed a commission to understand and study this ethical dilemma [150]. In June 2017, the commission proposed its final report that provides twenty ethical rules that all AVs must follow [151].

Generally, manufacturers and regulators will need to accomplish three potentially incompatible objectives: consistency, avoiding public outrage, and not discouraging buyers. One step toward solving this problem is trying to learn how people feel about alternative decisions that self-driving vehicles' AI might have to make. Thus, MIT lab created a platform called Moral Machine to allow the public to express their opinion on what actions should AVs choose in different scenarios [152]. The platform gathered the opinion of millions of people from 233 countries with 40 million decisions in ten languages. However, results show broad differences in relative preferences among different countries [153]. However, results show countries within close proximity to one another showed closer moral preferences, with three dominant clusters in the West, East, and South [154]. Generally, results of the moral machine show that people prefer sparing humans over animals, sparing more lives, and sparing young lives [153]. In general, results of the Moral Machine suggest that people want AVs to treat people unequally, preferentially killing some people over others. For example, results show that people prefer saving the lives of women, the young and the rich than the lives of men, the old, and the poor [155].

These results by contrast clash with many rules such as the IEEE code of ethics and some of the German ethical rules that clearly prohibit any distinction based on personal features such as age. However, results of the moral machine show that people strongly preferred sparing the young (such as children). These results do not mean that policymakers should adopt the public opinion. Given the strong preference for sparing children, policymakers must be aware of a dual challenge if they decide not to give a special status to children: the challenge of explaining the rationale for such a decision, and the challenge of handling the strong backlash that will inevitably occur the day an autonomous vehicle sacrifices children in a dilemma situation [153].
In another survey that was conducted on the US results shed light on the complexity of the decision-making process in dangerous situations. In this survey, respondents were asked whether the vehicle should save passengers or pedestrians. Generally, respondents approved that the vehicle should minimize the number of casualties. However, respondents show positive attitude towards saving the AV's passengers when they had to imagine themselves and another person, particularly a family member, in the AV [154].

Generally, previous surveys shed light on the social dilemma associated with AVs as follows:

- In 2015 and 2016, Bonnefon, et al. investigated the public acceptance of two possible AVs' crash algorithms: utilitarian (sparing more lives) and non-utilitarian (protect passengers of AVs). Respondents show a strong preference for the utilitarian algorithm (76\%), especially if passengers of the AV are not sacrificed. On the other hand, when respondents were asked for the likelihood of buying a car with a utilitarian algorithm, they show higher likelihood for purchasing vehicles with the nonutilitarian algorithm. Additionally, respondents stated that they do not agree that governments should enforce the utilitarian algorithm [156, 157]. The previous results indicate a social dilemma as people generally agree on what should be done for the greater good of everyone, but it is in everybody's self-interest not to do it themselves.

- In the study by Hohenberger respondents were asked to choose one of pre-specified car ethical settings. These ethical settings were based on one of four choices as follows: utilitarian, self-focused, random, or law-based approach. Results show that respondents do not accept the self-focused approach nor the utilitarian. Generally, respondents follow the law-based approach or the random approach. Thus, Hohenberger concluded that the protection of one's own life is not a priority for the participants and that users of AVs should have the possibility to choose ethical settings by decision guidance [158].

- Frison et al. used a simulator in order to understand how crash risks influence peoples' decisions in the crash. Participants had to choose between two options: swerve and kill pedestrians, or swerve and make a random crash, to avoid pedestrians, with a randomly determined probability of $0 \%, 25 \%, 50 \%$, or $75 \%$. Results show that respondents show no distinction between friends and strangers. Additionally, people are less likely to sacrifice children's lives. Additionally, many people will choose to sacrifice their own life to rescue others even if they have no survival chance and only $16 \%$ of the participants would sacrifice the pedestrians and save their own lives [159].

- In the experimental setup by Faulhaber et al. using virtual reality, participants drove manually along a street in a suburban area and two obstacles ahead appeared on 
the two lanes ahead of them and the participants had to decide which of the two they would save. Results show that people prefer the utilitarian approach and decided in favor of the (quantitative) greater good. Additionally, results show that almost half of the participants were willing to sacrifice themselves to save only one other road user [160].

Summarizing the results of the previous studies gives a similarly mixed picture as in the ethicists' discussions. There are findings in which respondents call a utilitarian approach for good, but also a selfish attitude can be found. Additionally, even those who in principle consider the utilitarian approach, are apparently not sure whether they want to sit in a car that sacrifices their lives to save a higher number of lives. In conclusion, one of the main problems facing AVs is that unlike human drivers, the decisions of AVs on how to crash are pre-defined by a programmer [148]. This is not only about ethics but also about the social acceptance of AVs that has a significant influence on the market penetration of AVs. Ethics and expectations are always challenging each other to all automotive stakeholders, so results can never satisfy everyone [143]. We find ourselves in a situation that is new to the world as vehicles will be able to make decisions on who should live and who should die with no real-time human supervision. This problem is not limited to a niche market but will affect everyday transportation and all road users, no matter whether they drive, walk, or ride a bike.

\subsection{Programming of the ethical decision making in autonomous vehicles}

The process of formulating the ethical rules in AVs faces two main challenges. The first one is how to define the value of the society across different scenarios. The second challenge is how to translate the set of ethical rules into a language that the vehicle can understand independently from any human intervention. From the academic perspective, previous studies can be categorized into one of three categories: rational approaches, artificial approaches, and hybrid rational and artificial intelligence approaches.

\subsubsection{Rational approaches}

The rational approach can be divided into two subcategories: deontological, and consequentialism. The deontological rules force the vehicle to follow a set of principles, while the consequentialism approach is an optimization approach that maximizes the overall benefits of the system. One well-know deontological in the domain is the Isaac Asimov's Three Laws of Robotics as follows: [161, 162]

- A robot should not be allowed to harm humans.
- A robot must obey human orders except where these orders conflict with the first law.

- A robot must protect its own exitance as long as this protection does not conflict with the first and second laws.

These laws can be translated for the case of AVs as follows [163]:

- An AV should not collide with a pedestrian or a cyclist.

- An AV should not collide with another vehicle unless this collision would not conflict with the first law.

- An AV should not collide with any other object in the environment unless avoiding such a collision does not conflict with the first and second laws.

The deontological approach can provide guidance in many scenarios, however, it cannot be considered as a complete ethical system because of the difficulty involved in the articulation of complex human ethics [148]. Additionally, this approach cannot address the problem related to the human factors in edge scenarios shown in a social dilemma, such as: would it matter if the pedestrian was child or was a pregnant woman [164]?

The other rational approach is called the consequentialism approach that analyzes the expected utility and evaluates a set of actions that maximizes the overall benefit. This approach has the potential to formulae the ethical decision making as an optimization problem [165] that maximizes the overall utility. For AVs, the utility can be defined as damage cost. However, this approach has its own shortcomings, such as the difficulty in defining the cost function or making it comprehensive and representative. Additionally, this approach will create new issues. For example, if the vehicle chooses between hitting a cyclist with a helmet and another one without a helmet, the vehicle will choose to hit the one with the helmet. This generates a new dilemma where those who paid or prepared for safety were unfairly targeted in emergency situations [166]. If this approach is followed no one would be willing to pay extra money to purchase a car with a better warranty or some motorcyclists might choose not to wear helmets. Thus, this approach might discourage people from following the safety procedures.

\subsubsection{Artificial intelligence approaches}

Since they have shortcomings, mostly due to the incompleteness to deal with the complex environment, alternative Artificial intelligence (AI) approaches have achieved much more success [167]. The main advantage of this approach is its ability to learn human ethics by rewarding the system's actions or observing human behavior [168]. However, the AI approach has its own shortcomings. First, the behavior learned by the AI approach cannot maximize overall safety 
on road due to the self-preservation instincts from a human. For instance, a human driver may intuitively decide to push another vehicle into an accident to avoid self-collision. Thus, the AI system should be designed carefully to capture ideal behaviors. The second shortcoming of the AI approach is the traceability [169]. Usually, artificial neural networks cannot explain how the decision was made based on the input data. The relationships are not easy for human brains to understand. The AV system designers should be cautious in implementing the AI approach. Since AI ethical method allows computers to mimic human ethics without human intervention to perform the difficult task of articulating ethics as computer code, they can not make sure the actions they take are not justified or explained in an understandable way. Even worse, it could learn behaviors that are completely undesirable if trained with a limited set of data.

\subsubsection{Hybrid rational and artificial intelligence approach}

As shown above, both the rational and AI approaches have their own shortcomings. Thus, a hybrid approach might represent a better solution. This hybrid approach adopts the machine learning method to study the human responses or actions on the basis of a wide range of real-world and hypothetical accident scenarios to formulate the ethical rules, meanwhile, the rules from the rational approach play the role of behavioral boundaries. This hybrid approach requires sophisticated software that does not exist yet, however, the neural network is likely a good candidate, which would be trained based on the data from recordings and simulations of crashes and near-crashes within a diverse set of training scenarios to promise the computer cannot learn an unintended ethical rule. To ensure reasonable decisions made by the vehicle, boundaries should be provided by rational approaches. To sum up, in this hybrid approach, the rule system from the rational approach remains in place as boundary requirements, and the AI method focuses on situations not covered by the rational ones [163]. Table 2 summarizes the different approaches used for ethical decisions in AVs.

\section{Liability and regulations, and implications on public acceptance}

\subsection{Liability and regulations for AVs}

Similar to accidents and ethical issues associated with AVs, liability, and regulations have a significant influence on public acceptance. One of the main challenges facing the introduction of $\mathrm{AVs}$ is the required changes in laws to fit AVs. Several regulations need to be adjusted to fit the AV technology such as the laws that state that every car must have a steering wheel, accelerator, or braking pedal [177].
The main issue is that most counties deal with the vehicle as a thing and the driver is the human who is liable in case of error or accident [178]. For example, the Vienna convention (1968) that was agreed by 74 countries states that drivers are always responsible for controlling the vehicle [30]. Therefore, the key question is: can AVs drive?

One of the proposed solutions is to define the AV software as the driver. However, this raises new concerns about liability, especially in complex situations $[179,180]$. One answer might be programming AVs with different ethical theories and let owners determine the vehicle's ethical values. In this approach, liability will be similar to liability nowadays and drivers will be liable in case of collision [178]. The software of $\mathrm{AVs}$ must be able to mimic human decisions, but some guidelines must be programmed to be ethical. As a result, the use of a black box might be mandatory to support the legal decisions. The German Ethical Guidelines (2017) stated that AVs must give priority to human life over animals or things [151]. They also stated that the AVs must be programmed to reduce the damage [30].

There are three types of liability: civil liability (damage to a third party), criminal liability (responsibility for death or injury), and administrative liability (driving with the absence of authorized requirements).

The administrative perspective is not significantly affected. However, the introduction of the AVs might change regulations. For example, a specific driving license might be required, or the insurance requirements might change. Laws will determine the circumstances of violations, so little concerns about the administrative aspect [48]. Moreover, there is a group of questions that need to be addressed about AVs in the administrative law such as: Can AVs operate on all roads or specific roads with separated lanes? Shall AVs follow the same traffic rules as traditional vehicles? [181].

Civil liability: According to the general liability laws, AV's occupant will not be responsible for the damage as he or she is not controlling the car similar to a bus passenger who is not liable in the case of collision. the manufacturer will be liable for errors in the final product (product liability). However, manufacturer liability might be limited if the manufacturer did everything to make the vehicles' owners aware of the defect and offer to remove it, but the owner did not take any action. On the other hand, AVs are trained using artificial intelligence (AI) and machine learning techniques, which makes it hard to identify the reason behind taking a specific decision. AI training involves millions of connections by means of training phase, which makes the process of tracking the decision at a specific moment a hard process and almost impossible [178].

In general, there might be two scenarios [182]. The first scenario is based on the driver liability and this approach is expected at the initial stage when both human drivers and AVs share the roads. In this scenario, the driver will be liable 


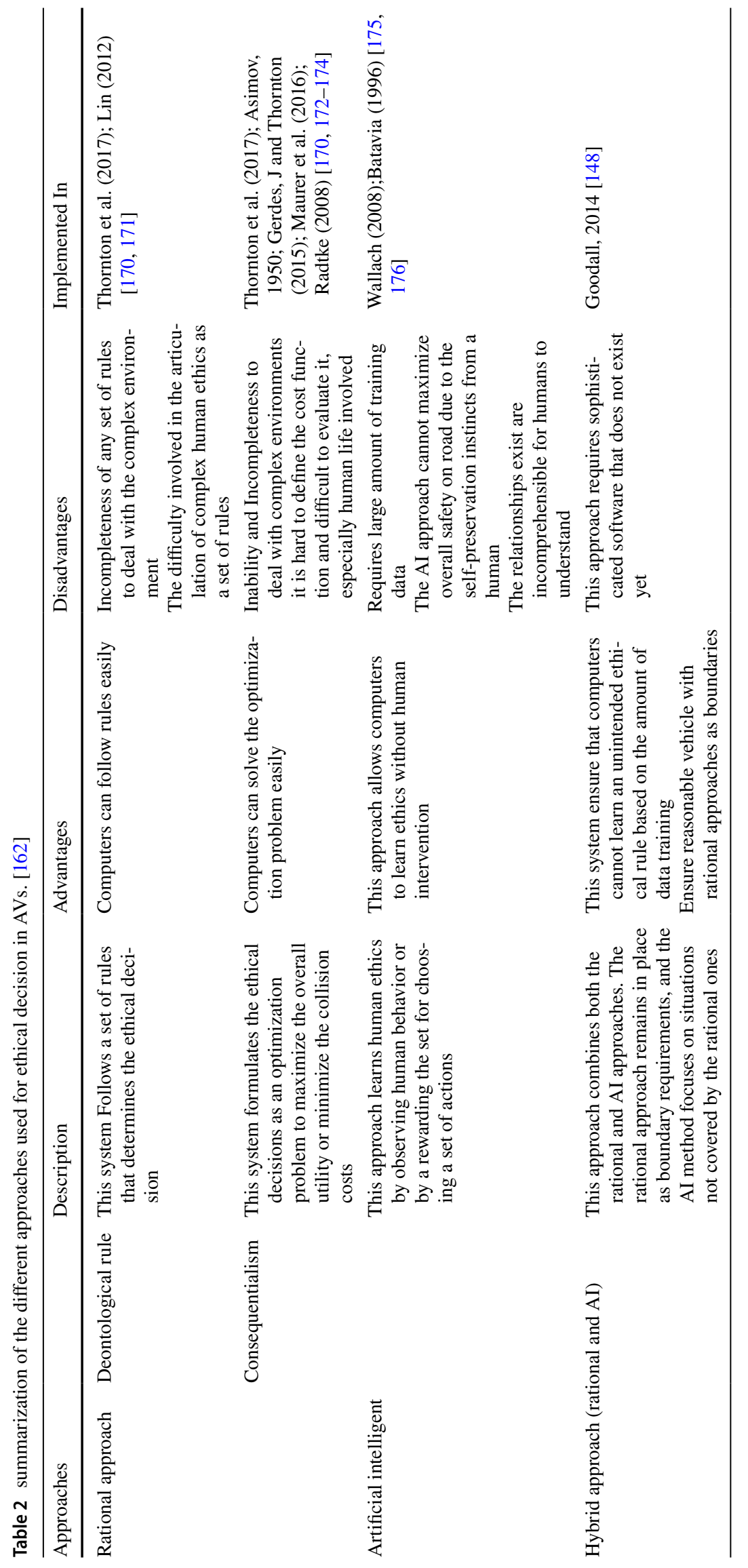


in case the vehicle provided him with sufficient warnings to take manual control and take an action to avoid collisions and with sufficient time to control. The second approach is expected to be adopted with high market penetration of $\mathrm{AVs}$ and it is based on manufacturer liability for defective products [178].

\subsection{Criminal liability and situational awareness dilemma}

Criminal liability is based on the strict responsibility of a crime. In the situation when no driver can be held responsible for the event, liability might be transferred to the manufacturer. However, the main question is whether the driver is still responsible in some situations or not. It is a sophisticated question as the driver might be oblivious (sleeping or reading). This motivates a new question "is the driver allowed to be oblivious during his trip?" or the diver is expected to be attentive and take control. Legally, there is no difference between the liability of the ordinary vehicle driver and AV's driver to the moment. As a result, it is possible to assume that it is not possible to exempt the driver from the liability at the moment. As AVs are in the initial state, the correct solution might be the driver liability. However, in the future with high penetration of AVs, it is expected that the driver liability will be mitigated. Possibly, a combination of the two extremes might be expected as the vehicle might include an alarm to ask the driver to take the manual control of the vehicle with enough time to react [178]. However, recent studies show that switching the control from the vehicle to the driver might be challenging because of the reduction in drivers; situational awareness. Situational awareness (SA) can be defined as the perception and comprehension of the surrounding information that allows a person to take the needed set of actions in response to the dynamic environment [183]. For the case of AVs, SA can be defined as the degree to which the is aware of the surrounding environment [184]. Poor situational awareness contributes to vehicle crashes as SA is a critical factor in drivers' ability in making decisions [185-187]. Safety of drivers demands an appropriate level of situation awareness, and this is of particular importance in autonomous vehicles for two reasons. First, the presence of the autonomous system will reduce drivers SA as the driving task will become a secondary task. Secondly, by definition, levels 2 and 3 of $\mathrm{AVs}$ require transitions between human driving and autonomous driving. As a result, it is extremely important for the safety of people that they can rapidly and comfortably reestablish awareness of the state of automation and awareness of the driving situation [188]. Maintaining sufficient alertness may be challenging for $\mathrm{AVs}$, as making sudden rises in cognitive demand is exceedingly challenging and potentially dangerous because with low attentional demand, cognitive resources may throttle back to conserve energy [189]. To quote Hancock's Automation Paradox "if you build systems where people are rarely required to respond, they will rarely respond when required" [190]. Previous studies applied a variety of research methodologies to understand the impact of vehicle automation on the SA, but simulators and questioners are the dominant methodologies used across the literature. Different studies show that increasing the level of automation reduces passengers SA as follows:

- One of the early studies that investigate the SA issue is the study by Endsley and Kiris. Results of this study showed that increasing the level of automation reduces the SA level from $98 \%$ (in manual driven vehicles) to almost 75\% (in fully AVs) [191].

- Behavioral data from Miller, et al. study that was conducted using a simulator show significant differences in time to initiate evasive action across conditions. Additionally, participants in fully AVs show greater comfort and trust in the system than partially AVs. Thus, the required time for drivers in fully AVs to take control increases [192].

- Behavioral data from Sirkin, et al. study that was conducted using a simulator to investigate the appropriate method to alert people to take control of the vehicle. Results show that $58 \%$ of respondents could correctly differentiate between different events in case of visible alert, $83 \%$ in case of audible alert, and $95 \%$ in the case of visible and audible alerts. Additionally, respondents need an average response time of 5-9 $\mathrm{s}$ in order to take control back depending on the alert method [193].

- Lin, et al. investigated the behavioral adaptations of early partially AVs' adopters after short-term usage. Interviews were conducted with $20 \mathrm{~T}$ drivers who have high experience (at least one to five months) with the autopilot mode. Results showed that partially AVs' drivers had a very positive attitude towards AVs, reported that they frequently got engaged in secondary activities. The manufacturer requests that Tesla owners keep their hands on the wheel in order to avoid abuse of the Autopilot and to ensure that they can take over immediately in critical situations [194, 195]. Although reports of engaging in potentially distracting activities were highly prevalent, all respondents stated that they already know that getting engaged in a secondary task was contrary to the instructions. Respondents stated that the time associated with a secondary task engagement, when eyes are off the road, is between 3 and $5 \mathrm{~s}$ and this time becomes longer when they drive on familiar roadways. $30 \%$ of the respondents stated that they frequently keep one hand on the phone and the other hand on the steering wheels. One of the twenty respondents stated that one time he spent half an 
hour playing on his phone without looking into the road [196].

- Behavioral data from Sun, et al. study that was conducted using a simulator to investigate the factors that influence trust levels on AVs shows that on average, participants had a higher level of situational awareness during manual driving, compared with those in AVs [197].

Thus, establishing a safe transition from the autonomous system to the human-driven system increase the complexity of the system. This complexity requires specific regulations to force people and manufacturers to comply with prespecified safety procedures such as forcing people to keep their hands on the steering wheels and forcing manufacturers to provide the appropriate alert to hand over the vehicle control safely.

\subsection{Impact of liability on the public acceptance}

While the mentioned liability issue is expected to influence the public acceptance of $\mathrm{AVs}$, rare studies endeavored to understand this phenomenon and how it impacts the public acceptance. As discussed before the survey study by Casley et al. was conducted in the US with 467 respondents and with the aim of understanding the impact of three factors on the public acceptance of AVs. These three factors are the safety of the system, the cost of the technology, and the liability issue [31]. The following bullets are the main questions asked in the survey and their results:

- Respondents were asked to rank the importance of safety, costs, and laws on their perception of AVs. 82\% of the respondents ranked safety as the most important aspect in order to adopt AVs, $12 \%$ believe that laws are the most important aspect, and $6 \%$ choose costs. These results show that safety is a top priority for people and illustrate that people will not adopt AVs until they make sure that this new technology is safe. Additionally, laws are ranked as the second factor that influences the public acceptance towards AVs.

- Respondents were asked if they would be comfortable sending their cars out on an errand by itself knowing that I am liable if it gets into an accident. The majority of respondents were not comfortable with the idea of being liable for an accident the car gets in if they were not driving it. $72 \%$ of the respondents were not comfortable with being liable, and $10 \%$ were comfortable with this idea, while $18 \%$ were in the neutral state.

- Respondents were asked the following question "By law, if a car's autonomous system fails the car is required to alert the driver and either give the driver control or pull over and come to a stop. I am comfortable knowing that this is required by law." The majority of respondents were comfortable knowing that the car will alert the driver, pull over, and stop if the autonomous system fails.

- Respondents were asked if a specific driving license should be required in order to legally operate an AV. Results show that $80 \%$ of people believe that a driving license should be required, $10 \%$ are in the neutral state, and $10 \%$ believe that no license is required.

- Respondents were asked "how do the laws concerning autonomous cars influence your desire to purchase one?". $10 \%$ of the respondents believe that laws will not impact their desire to purchase an $\mathrm{AV}, 50 \%$ were in the neutral state, and $40 \%$ believe that laws will affect their desire in adopting AVs. These results show how liability can influence the public attitude towards AVs and that a large proportion of respondents might not adopt AVs for policy reasons. It must be mentioned that $50 \%$ of the respondents are in the neutral state which indicates the low level of awareness regarding AVs' laws.

A second survey by Rezaei, and Caulfield show that people are not at all willing to accept liability for AVs. Results show that almost $60 \%$ of the respondents believe they must not be liable in case of accident, while only $14 \%$ believe they should be liable. Additionally, $71 \%$ of the respondents believe that AVs' manufacturers should be the liable agency in case of accident, while $22 \%$ believe that insurance companies should be liable [198].

Different other surveys all over the world and across the years show that people have high levels of concerns regarding the liability of AVs as follows:

- The survey by Richardson et al. shows that $60 \%$ of the respondents are highly concerned about the liability of the AV in case of accident [34].

- The survey by Piao et al. in France shows that 56\% of the respondents are highly concerned about the liability of the AV in case of accident [199].

- The survey by Cunningham, Ledger, and Regan in Australia and New Zealand shows that $84 \%$ of the respondents are highly concerned about the liability of the AV in case of accident [200].

- The survey by Greaves et al. in Australia shows that 64\% of the respondents are highly concerned about the liability of the AV in case of accident [37].

Also, AVs will face new issues related to cybercrime and hacking. The criminal law will have to address new questions such as: who should be responsible if an AV is used in a crime? As incidents happen in different environments, will the responsible subject change depend on the circumstances? [181].

For example, in 2016, a team of researchers hacked a Tesla car and took control of the car for almost 12 miles. 
The Chinese researchers were able to control every electronic feature of the vehicle remotely such as brakes and car locking. The attack requires the car to be connected to a malicious Wi-Fi system set by the team and the attack can be done only if the car browser is opened. Tesla stated that the probabilities to meet the attack requirements were very low, but this would not stop them from responding quickly [201]. In fact, cybersecurity represents a major concern for the public. Surveys showed high levels of concerns regarding security as follows:

- $90.9 \%$ of respondents are concerned about vehicle security [34]

- $54 \%$ of respondents are concerned about vehicle security [199]

- $68 \%$ of respondents are concerned about vehicle security [37]

As a result, vehicle security is an important feature to gain customers' trust.

In conclusion, there is a critical issue in the liability between human drivers and manufacturers that will be raised soon. Future legislations must limit the liability of passengers in case of inattentive, especially the main benefit of $\mathrm{AVs}$ is to allow passengers to be involved in other activities during their trips. Thus, if the driver is asked to keep attention on the road while the car is moving, then what is the difference between the AVs and the conventional vehicle?! In such a case, will people accept to buy such a vehicle?! Unfortunately, in general, governments are following the principle of blame avoidance because of the safety concerns, which hinder the technology and increase the costs [202]. Also, the legal sector is following the development of AVs instead of taking the lead [178].

Given this, autonomous car manufacturers should emphasize the safety of autonomous cars and prove to the public that operating an autonomous car is not a risky endeavor (as discussed on the safety Sect. 3). These manufacturers must also push to have thorough and reasonable laws for autonomous cars developed. Only when the cars have been made safe and the laws surrounding them made legally satisfactory the people will judge the cost of these cars to be affordable or not. Until these concerns are met, the price of the vehicle is inconsequential to the purchase of the vehicle.

\section{Public acceptance and perception of the AV technology}

As discussed in the previous sections, there are many factors that influence the public acceptance of AVs' technology and might push people from adopting this technology. This section discusses in detail the public perception of $\mathrm{AVs}$ to reveal the main factors that might motivate or discourage people from adopting AVs.

\subsection{Impact of previous experience (awareness)}

Piao et al. evaluated the public opinion about AVs with the aim of understanding the impact of the previous experience with AVs technology on public acceptance of the technology using an online survey and telephone interview in La Rochelle, France because six automated buses piloted in the city in 2015 [199]. Results of the survey imply the following:

- $87 \%$ of the survey have previous experience with AVs.

- Majority of respondents were optimistic about the AV technology with $50 \%$ believe that AVs reduce energy and emissions.

- $2 / 3$ of respondents prefer autonomous buses than humandriven buses because of the cost reduction due to no driver costs.

- $73 \%$ of people with previous experience with AVs prefer trips on AVs compared with 55\% for respondents without previous experience. Thus, previous experience with AVs has a significant influence on the public acceptance.

Simulators can provide a second approach to evaluate the impact of previous experience. Consequently, Wintersberger et al. used a driving simulator to study the user acceptance with 48 participants riding an AV with positive and negative affect schedule (PANAS), affect grid [two-dimensional grid measuring pleasure against sleepiness to arousal], interviews. and questionnaires that were conducted twice; one before and another after the trip to analyze the participants' emotions. The experimental setup attempt to answer the following question "Do people accept an AVs the same way as they accept human drivers", by testing the following hypotheses: "There is no difference in the mental condition nor the emotional state of front-seat passengers of an $\mathrm{AV}$, male or female driver" [203]. Overall conclusion, respondents who had previous background in AVs were more optimistic about it.

On the other hand, Richardson and Davies created an online survey in UK with 199 respondents then compared the results with a similar survey concluded earlier in 2014 to understand the change in public opinion over time. Results showed that: in 2017, there was a negative shift in the public opinion about AVs, when compared to 2014 results. Although more people became more aware of AVs in 2017, the percentage of respondents with positive opinions dropped by $25 \%$ in the 3 years [204]. Results of this survey comply with the results from Fig. 3 as in 2014 there were no $\mathrm{AVs}$ available and there were no accidents, so people were optimistic about AVs, however, with time AVs' accidents 
are reported and people became more concerned and less positive towards AVs.

\subsection{Impact of economic conditions on public acceptance of AVs}

Bazilinskyy et al. (2015) conducted three surveys in 112 countries with 8862 respondents to estimate the public acceptance in the national level. Results revealed that people from low-income countries are more likely positive towards AVs than people from high-income countries. $40 \%$, $20 \%$, and $23 \%$ of respondents from high, medium, and lowincome countries were concerned about the AV technology. Respondents from high-income countries were concerned about their software failure. Additionally, developed countries were less comfortable with the idea of vehicle transmitting data than developing countries [205].

\subsection{Truck drivers and fleet operators perception of AVs}

Although truck drivers and freight industry are expected to get affected significantly with the introduction of AVs, rare studies focused on the perception of truck drivers and fleet operators. Consequently, it is essential to understand the opinion of truck drivers and fleet managers on AVs. Richardson et al. conducted an online and in paper survey for 69 truck drivers and 17 fleet managers [34]. Results showed that:

- Significant portion of the truck drivers (44\%) are not aware of the AV technology, while $90 \%$ of fleet managers already have the knowledge about the new technology.

- $74 \%$ of truck drivers feel safe about their jobs.

- $51 \%$ of the truck drivers believe that AVs might increase traffic safety and comfort.

- $47 \%$ of truck drivers choose their job because of driving pleasure.

- Privacy and liability of AVs are the major sources of fleet managers and truck driver concerns, and a significant portion of truck drivers (46.2\%) are concerned about the loss of driving pleasure.

A significant portion of truck drivers feels safe regarding their jobs. Maybe because they are not aware of the new technology or they believe that AVs will not replace them. Actually, a recent study by Gittleman and Monaco revealed that although the risk on truck drivers is real, the projections touted are exaggerated because companies will always need drivers because not all the driver's tasks can be automated. For example, sensors can detect safety problems, but they cannot do anything about them, so fixing these problems requires human interaction [206].

\subsection{Willingness to pay}

Willingness to pay is a key factor for the success of any new technology, especially in the initial state where the cost of the new technology is high. Previous surveys show that a small proportion of people are willing to pay more for AVs as follows:

- Schoettle and Sivak found that almost $60 \%$ of respondents in the US, UK, and Australia are not willing to pay more for the new technology, while only $10 \%$ are willing to pay much more [34].

- Kyriakidis et al. found that only $5 \%$ of respondents are willing to pay more than $\$ 30,000$ for an AV. Additionally, results showed that AVs are more attractive to people who make long trips and to people who live in countries that register high accident rates [36].

- Cunningham et al. found that $66 \%$ and $57 \%$ of respondents are not willing to pay more for AVs [200].

In summary, a small proportion of the respondents are willing to pay more for AVs [26].

\subsection{Precepting of AVs for different age groups}

Surveys showed that younger people are more enthusiastic towards AVs' technology as follows:

- Piao et al., found that $56 \%$ of respondents aged $>65$ would consider making trips using AVs, compared to $62 \%$ and $61 \%$ for people aged between 18 and 34, and 35-64 [199].

- Abraham et al., found that $40 \%$ of the 25-34 years old participants prefer AVs, while only $12 \%$ for 65-74 years old consider making trips in AVs [207].

- Richardson and Davies found that people become discouraged about AVs with the increase in the number of years driving [204].

While many studies assume that AVs have the potential to increase accessibility for aged individuals and consider this segment as the early adaptor of AVs [19, 208-211], results show that younger people are more interested in AVs, which contradict with the theory that elder will benefit more from AVs.

Additionally, while the disabled are considered as one of the early adaptors of AVs, no study survived this group to estimate their acceptance of this new technology. As a result, further surveys are required to understand the disabled perception of the new technology instead of making assumptions similar to the elder adaption theory. 


\subsection{Perception of males and females}

Surveys showed that males are always more optimistic towards AVs than females as follows:

- Schoettle and Sivak, illustrated that males are more positive toward AVs' adoption [34].

- Schoettle and Sivak, found that males are more interested in AVs, with $19 \%$ of the males fully AVs compared to $12.4 \%$ of the females. Additionally, females responded with higher levels of concern than males as $30 \%$ of males were concerned about fully AVs compared to $40 \%$ of females [35].

- Piao et al., found that males are likely to use AVs than females. Of the male respondents, $64 \%$ have no problem in making trips in AVs, compared to 55\% females [199].

- Abraham et al., found that males were comfortable with higher levels of automation with $53 \%$ of the males have no problem allowing the vehicle to take control compared with $40 \%$ of females [207].

- Richardson and Davies: females are more concerned about the risks of AVs than males, with an average 3/5 of the males believe that AVs will increase the safety compared with $2.37 / 5$ of the females [204].

\subsection{Impact of educational level on AVs' acceptance}

Higher education people are more aware of AVs' benefits and concerns and generally skeptical [34]. Results of Piao et al., study showed that people with higher educational levels are more positive towards AVs. $71 \%$ of high education respondents stated that they prefer AVs, compared to 52\% for people with low education [199].

\subsection{Public preference of different modes of AVs (public vs. private) and the cost of technology}

Affordability is a major concern facing the introduction of AVs. For example, according to the National Automobile Dealers Association, Americans spend an average of 30,000 $\$$ for buying a new car. However, the cost of an $\mathrm{AV}$ is almost $322,000 \$$ because of the Velodyne LIDAR system, visual and radar sensors, and the cost of the driving computer and software [212, 213]. Autonomous taxis can circumvent this cost issue. In 2019, Nunes and Hernandez studied the cost competitiveness of autonomous taxis when compared to conventional vehicles. The study went through four main phases: first, estimated the cost of owning an older vehicle; second, use the public data to estimate the operating costs of taxis; third, adjust expenses to include the new technology; finally, econometric testing. Results indicate that autonomous taxis can cost customers up to three times more than conventional vehicles [213].
On the other hand, the US department of transportation (2017) conducted an economic analysis that considered the current technology, the associated ownership, operating costs, and compared them to the estimates in the future. Results indicate that AVs have the potential to reduce the operating costs by 30 to 50 cents per mile [214]. Similarly, Ongel et al. conducted an economic analysis that indicates that autonomous taxis can reduce the operating costs by 60 to $75 \%$ compared to conventional vehicles [215]. Todd Litman provided a comparison between the costs of traditional driving vs. AVs in different modes. Results showed that operating costs of private AVs will be cheaper than traditional taxis but higher than the traditional transit and traditional vehicles. However, shared AVs will be cheaper than any other mode [216]. Figure 7 shows a cost comparison for the different modes of $\mathrm{AVs}$ and conventional vehicles.

While shared AVs have the potential to reduce the operating costs, it cannot provide high service quality. Consequently, it is expected that most of the surface will be stainless steel and plastic to minimize the cleaning and vandalism costs. Additionally, passengers will be monitored by security cameras. Shared AVs will be similar to public transportation modes where people share their private space, encounter previous occupants' garbage, and there will be no driver to help passengers. Additionally, passengers might encounter additional delays because of the pickup and drop of events. Thus, private AV might be the preferred mode for many people [216]. Thus, it is essential to investigate the public preference of different AVs' mode (public, private).

Piao et al. survey in France showed that $67 \%$ of respondents preferred autonomous buses over human-driven buses because of the cost reduction due to the reduction of the driver costs. On the other hand, $33 \%$ of respondents preferred autonomous taxis over conventional taxis. It must be mentioned that $33 \%$ of the respondents stated that they do not know which mode they prefer because they are no familiar with the sharing service provided by AVs [199]. In 2018, Moreno et al. survey in German showed that $42 \%$ of the respondents are willing to share their trips while using $\mathrm{AVs}$ [217]. However, $23 \%$ of the overall trips in Germany are made using a shared mode [218]. As a result, AVs have the potential to shift the personal transportation from personal use to shared use.

However, it must be mentioned that findings of the previous surveys are not guaranteed, because highly or fully autonomous and connected vehicles are not currently available on the market. Thus, results of the previous surveys rely on the imagination of the respondents regarding the operation of AVs in the future. Thus, over time communities and individuals learn more about this new technology, and their perceptions, expectations, and stated behavioral responses are likely to change, in some cases rapidly. Consequently, 
Fig. 7 Cost comparison between the different modes of $\mathrm{AVs}$ and conventional vehicles [216]

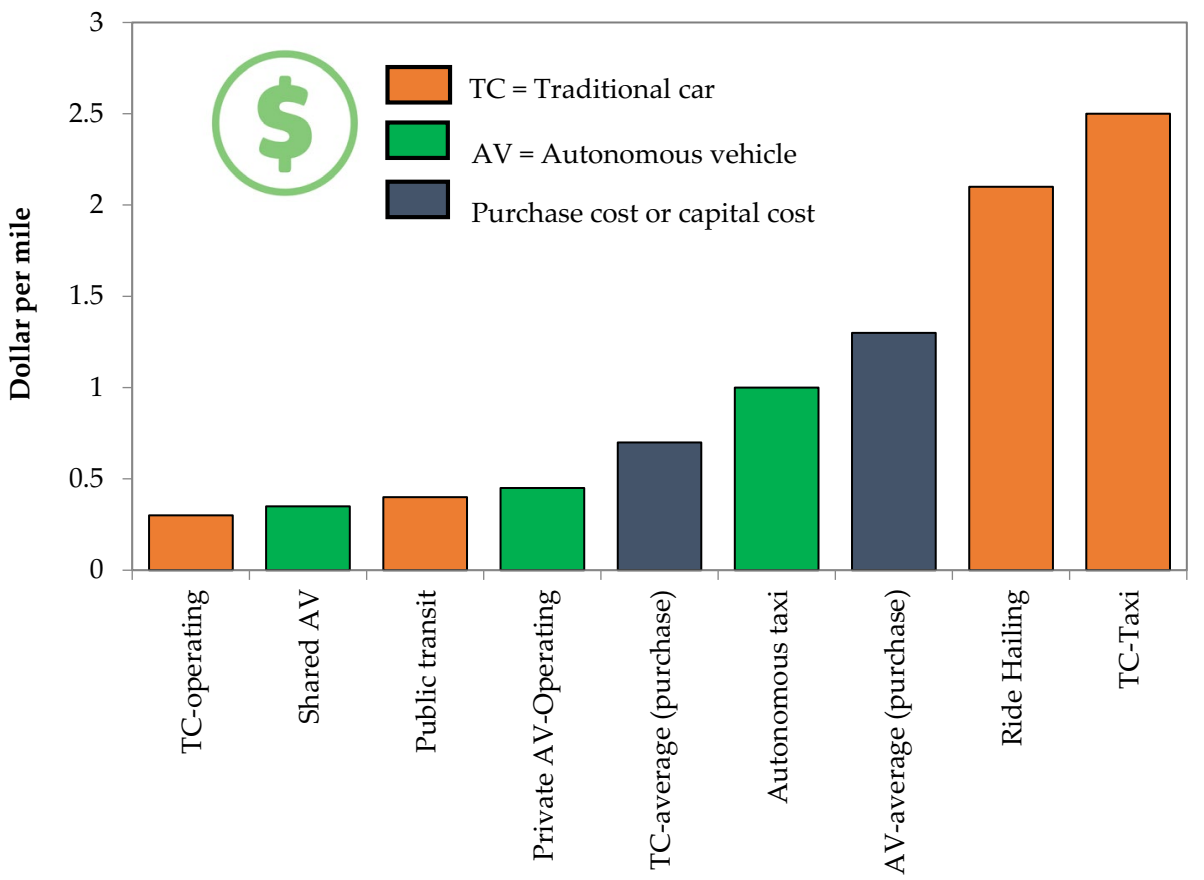

the public preference for different modes of AVs must be investigated over time.

Figure 8 provides a statistical summary of the public perception covered in this paper. The figure shows the minimum, maximum, and average percentages of the different attributes covered in this paper. In general, males are more positive towards AVs than females. Young and midage groups are more positive towards AVs than the older age group. A significant portion of people are not willing to pay more for $\mathrm{AVs}$, and a small proportion is willing to pay much more. People from low GDP countries are more positive towards AVs than people from medium and high
Fig. 8 Summary of the of the public perception of AVs

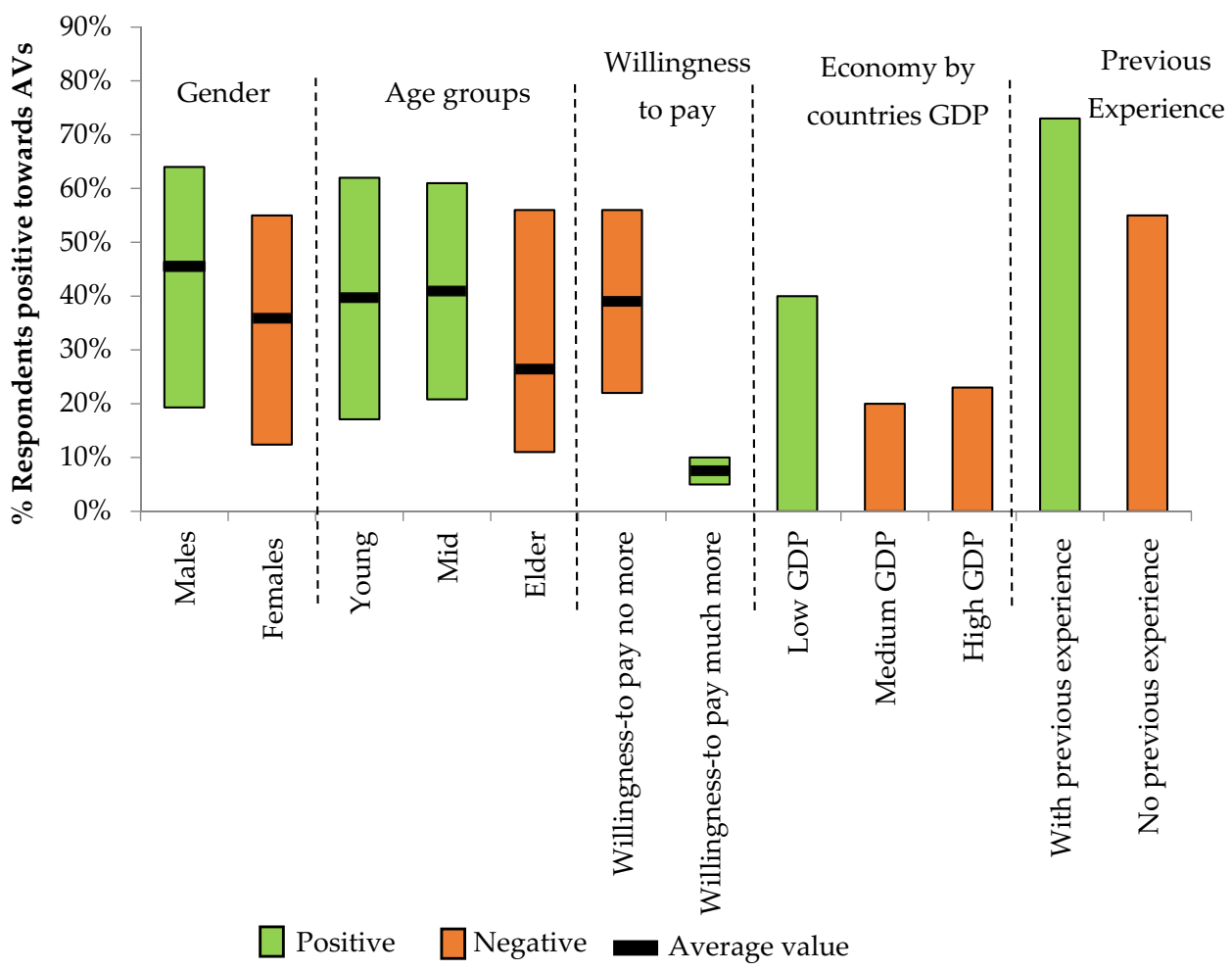


GDP countries. Finally, people with previous experience with AVs are more positive towards AVs than people with no experience.

\section{AVs in pandemics and implications on the public attitude towards AVs}

The global COVID-19 has made radical changes in our world, as people had to adapt to a new lifestyle. In the last few months, public health experts advised people to avoid crowds, enclosed spaces, and time spent in close contact with others, which are normal features of the transit system. The level of fear of public transit service increases significantly after this pandemic as many people blame subways and buses for coronavirus outbreaks [219]. For example, it was found that ridership on bus and rail systems in the USA had dropped by $74 \%$ in New York, $79 \%$ in Washington, D.C., and $83 \%$ in Boston. In Canada, transit ridership dropped by $80 \%$ in Toronto and $82 \%$ in Montréal [220]. Additionally, one study in New York City entitled "The Subways Seeded the Massive Coronavirus Epidemic in New York City" by an MIT economics professor shows that subways are the major source for the widespread of the virus [221] that some of the city officials requested the governor to shut down the New York's transit system [222]. In Ontario, Canada, the province asked the TTC to consider replacing some of their bus routes with microtransit service through partnerships with companies like Uber [223]. Thus, it is essential to look for an alternative for the public transportation service (buses, subways, etc.) to fill this gap and at the same time maintain equity. This alternative must be affordable to everyone and provide a service similar to the public transportation service. As mentioned above in section (7.8 Public preference of the mode of AVs (public vs. private) and the cost of technology:), AVs might be the future alternative for the public transportation service. Previous AVs' simulation models show that AVs as a shared mode (similar to Uber- the vehicle is shared between customers, but it serves one customer at a time) can provide the same mobility with low trip costs when compared to human-driven vehicles or human-driven taxis as follows:

- A simulation model for Ann Arbor, Michigan, USA to achieve a customer waiting time of two minutes or lower shows high-cost reduction from 21 \$ to $2 \$$ (90\% reduction) per day due to reduction in the ownership cost, operating expenses, parking fees and value of time [224].

- Two simulation models for Babcock Ranch, Florida, USA and Manhattan, New York, USA showed radically low trip cost with an average waiting time less than two minutes. In Manhattan, results show high-cost reduction from $7.8 \$$ per trip (using the traditional yellow taxi) to
0.8 \$ per trip (88\% reduction) due to the reduction in the ownership cost, operating expenses, and central coordination. For the Babcock Ranch case, the mobility service cost would be less than $3 \$$ per day per person or $1 \$$ per trip [224].

- The US department of transportation conducted an economic analysis that considered the current technology, the associated ownership, operating costs, and compared them to the estimates in the future. Results indicate that $\mathrm{AVs}$ have the potential to reduce the operating costs by 30 to 50 cents per mile [225].

- Ongel et al. conducted an economic analysis that indicates that autonomous taxis can reduce the operating costs by 60 to $75 \%$ compared to conventional vehicles [215].

- Litman provided a comparison between the costs of traditional driving vs. AVs in different modes. Results show that operating costs of private AVs will be cheaper than traditional taxis but higher than the traditional transit and traditional vehicles. However, shared AVs will be cheaper than any other mode [216].

From the previous discussion, it is shown that AVs can provide the same mobility with low trip costs. This cost reduction guarantees equity and affordability of this service to everyone. Therefore, experts believe that this pandemic is a turning point that will accelerate the new digital revolution. Although the pandemic has halted many AVs' pilot studies $[215,226]$, it is expected that this crisis will accelerate the introduction of AVs as AVs can be useful in emergencies and pandemics as follows:

- AVs have already proven their value in the times of pandemics. China used autonomous vans for food and medical supplies delivery and sanitize streets [227]. For example, Apollo has partnered with Neolix for the delivery of food and medical supplies in Beijing [228]. Additionally, in Florida, the Mayo Clinic has started using the AV developed by Beep to transport COVID-19 tests from the testing site to the processing laboratory [229]. In the future, during pandemics, AVs can be used as a transportation mean to transport people to grocery stores, healthcare, and pharmacies, while maintaining isolation and sterilization [226, 227].

- Start-up companies such as Vayyar are developing vehicles for monitoring vehicle cleanliness and air quality that measure airborne contaminations for infected passengers. This feature can be useful for the early detection of diseases [227].

- More importantly, AVs could replace public transportation in pandemics which is a main source for the spread of diseases. For example, in 2018, Goscé and Johansson found a correlation between the use of public transport 
and the spread of diseases in London. Additionally, it was found that underground transportation plays a significant role in the spread of airborne infections [230].

$\mathrm{AVs}$ have proven their ability in addressing some of the biggest challenges confronting society in pandemics. AVs can provide effective, safe mobility to help people to move to their essential activities. Although the use of AVs is still uncertain, as well as it needs a long time in terms of the regulatory process, the previous discussion, and the mentioned use cases above have proven the worth of autonomous vehicles that makes AVs a major tool in the fight against pandemics. Consequently, AVs have the potential to become part of our daily life in a post COVID-19 world. As a result, it is expected that this pandemic will have a significant influence on the public acceptance of $\mathrm{AVs}$ and people might become more optimistic towards AVs.

A recent survey by The Motional Consumer Mobility in the US shows that the public enthusiasm in AVs is growing [231]. The Motional Consumer Mobility Report surveyed 1,003 U.S. consumers in the mid of July to find public enthusiasm toward driverless vehicles is generally growing [232]. Results show that:

- COVID-19 has redefined the definition of safety. $70 \%$ of the respondents agree that COVID-19 has changed how cities should be planned in the future.

- $83 \%$ of the respondents agree that access to safe, clean transportation is a public health issue.

- $70 \%$ of respondents admit that the risk of infection is a real concern impacting their transportation decisions.

- $60 \%$ of the respondents are reconsidering their transportation choices to accommodate social distancing.

- $86 \%$ of the respondent will travel in an AV if they are given the opportunity.

- $66 \%$ of the respondents will consider using AVs regularly.

- $82 \%$ believe that AVs are the way to the future.

Result of this survey shows that the level of awareness, and interest in autonomous systems in general, increases while people are facing this new world as they can not necessarily do things in the same way that they did in the past. Additionally, results of this survey show significant increase in the level of interest in AVs in the US when compared with another survey that was conducted by Partners for Automated Vehicle Education (PAVE) just before the widespread effects of COVID-19 hit the U.S [233]. PAVE survey showed that $48 \%$ of the Americans would never consider an AV for their trip. Thus, this pandemic has caused conversations around autonomous driving systems to happen more broadly than before, which has opened consumers' eyes to the benefits of AVs [232].
The ongoing COVID-19 pandemic has created a surge in the public interest and demand for autonomous delivery robots since it can provide contactless delivery as discussed above. Thus, another study for evaluating the public acceptance of autonomous delivery robots during COVID-19 pandemic was conducted in Portland, US with 483 respondents. This study focuses on the consumers' willingness to pay for this emerging technology. Results show that $62 \%$ of respondents are willing to pay more than $2.3 \$$ to receive deliveries using autonomous delivery robots or AVs [234].

\section{Conclusions}

Since the development of the first AV by Mercedes-Benz and Bundeswehr University in Munich, tremendous efforts have been dedicated to AVs. While AVs are often discussed in regard to their implications, benefits, technological development, and technological challenges, less attention has been paid to the public acceptance and perception of AVs. Thus, This paper reviews the previous studies that focused on testing the public acceptance and perception of $\mathrm{AVs}$ and sketches out the main trends in this area with the focus on implications of safety, ethics, liability, and regulations on the public acceptance. This paper shed light on the main trends and factors influencing the public perception and acceptance of $\mathrm{AVs}$ as follows:

- While AVs have the potential to increase traffic safety due to the avoidance of the human error, vehicular failure might replace the human error due to many reasons such as the variety of situations or scenarios AVs might be involved in and the variety of the environmental conditions. These reasons make the process of training AVs a highly sophisticated process that requires a high level of cooperation and coordination between multiple disciplines from machine learning, to sensor development, to AVs' testing and developing a standard testing process

- In general, people are highly concerned about AVs, and the level of fear of AVs increase with the increase in the number of accidents reported.

- AVs faces an ethical dilemma similar to the famous trolley paradox, however, the paradox will be more frequent for the case of AVs. The main issue is that unlike human drivers, AVs' decision on how to crash is pre-defined by a programmer. This dilemma has a significant influence on the public acceptance of AVs.

- There is a critical issue in the liability between human driving and manufacturer that will be raised soon. The future legislations must limit the liability of passengers, otherwise, if drivers are forced to keep attention on the road while the car is moving, then what is the difference between $\mathrm{AVs}$ and conventional vehicles?! In 
such a case, will people accept to buy such a vehicle?! Apparently, the legal sector is following the development of AVs instead of taking the lead.

- Previous experience with AVs has a significant influence on the public acceptance of AVs. People with previous experience with AVs' features are more positive towards adopting AVs. However, it must be mentioned that previous experience might affect the public acceptance negatively as discussed in Richardson and Davies (2018) study [204] because the public acceptance is affected by other factors such as AVs' accidents.

- While truck drivers are expected to suffer from job loss with the introduction of $\mathrm{AVs}$, rare studies focused on the perception or the opinion of truck drivers on AVs' technology. Nevertheless, a recent study by Gittleman and Monaco (2020) revealed that although the risk on truck drivers is real, the projections touted are exaggerated because companies will always need drivers as some of the driver tasks cannot be automated. While sensors can detect safety problems, they cannot do anything about them, so fixing these problems requires some human interaction [206].

- While the disabled are considered to be one of the early adaptors of AVs as AVs increase their accessibility, no study surveyed this group to understand their acceptance and perception of the AV technology. Similarly, the elders are considered one of the early adaptors of $\mathrm{AVs}$ as AVs will increase their accessibility. However, surveys showed that older people are the most pessimistic towards AVs, which contradicts the theories that the elder will benefit more from the AVs.

- While willingness to pay is a key factor for the success of any new technology, especially in the initial state where the cost of the new technology is high, previous surveys show that a small proportion of people are willing to pay more for AVs.

- Males are more positive towards AVs than females. Similarly, people with high education levels are more positive than people with lower education levels.

- AVs have the potential to help people in their fights against pandemics. AVs can be used to transport people while maintaining isolation and sterilization. Additionally, AVs can be used for the delivery of food and medical supplies. Thus, the COVID-19 pandemic has caused conversations around autonomous driving systems to happen more broadly than before, which has opened consumers' eyes to the benefits of AVs and in turn has increased the public enthusiasm towards AVs.

Figure 9 summarizes the main conclusions and outcomes of this study.

\section{Final remarks and recommendations for the future}

- Safety: the safety of autonomous cars is paramount. If the vehicles are not safe, they are significantly less desirable, regardless of their benefits. The perceived safety, or rather the perceived lack of safety of autonomous cars, is what will truly sway the opinions of potential buyers. Given this, autonomous car manufacturers should emphasize the safety of autonomous cars and prove to the public that operating an autonomous car is not a risky endeavour.

- Liability: AVs' manufacturers must also push to have thorough and reasonable laws for autonomous cars developed. Only when the cars have been made safe and the laws surrounding them made legally satisfactory the people will judge the cost of these cars to be affordable or not. Until these concerns are met, the price of the vehicle is inconsequential to the purchase of the vehicle.

- Ethical dilemma: one of the main problems in the AVs is that unlike the human driving, AVs' decision on how to crash is pre-defined by a programmer. These decisions have a great influence on public acceptance of AVs. This issue is similar to the Trolley paradox; however, for AVs the dilemma seems much more serious as AVs will be involved in this dilemma much more frequently than a train running in a railway. Further studies are required to understand the appropriate decision in similar situations and understand the social acceptance of different alternatives.

- Target segment: AVs' providers should target young, educated males as this segment is the most attracted to AVs' technology. Probably AVs' provider should train their vehicles in universities to familiarize this segment with the new technology. However, this segment cannot pay much for this technology so the use of shared AVs might be a good and affordable solution. For example, autonomous shuttles can be used as a first-mile and last-mile solution between transit stations to universities.

- Elder and disabled debate: AVs are expected to increase the accessibility for elders and the disabled. While the disabled are considered to be one of the early adaptors of the AVs, no study surveyed this group to understand their acceptance of the AVs. As a result, future studies are required for this group instead of making assumptions. Similarly, elders are considered one of the early adaptors of $\mathrm{AVs}$ as $\mathrm{AVs}$ will increase their accessibility. However, surveys showed that the elder is the most pessimistic segment towards AVs, which contradicts the theories that the elder will benefit more from AVs. 


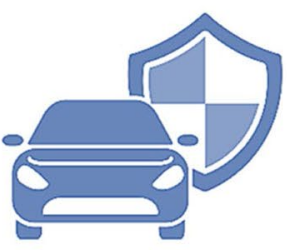

AVs eliminate the human error, but vehicular failure will become more frequent

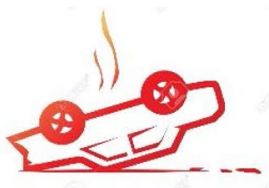

AVs accidents increase the public fear of AVs

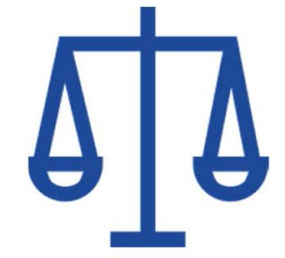

The future legislations must limit the liability of passengers, or else people will not accept AVs.

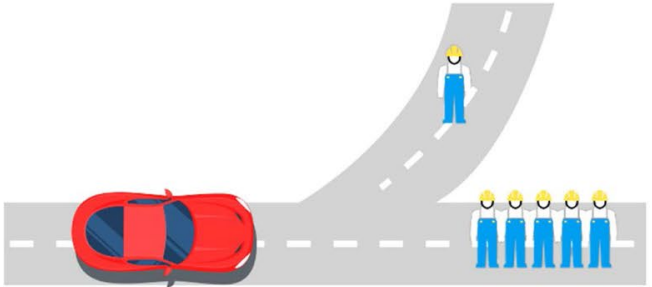

$\mathrm{AVs}$ faces and ethical dilemma as AVs decision on how to crash is pre-defined by a programmer, which has a significant influence in the public acceptance.

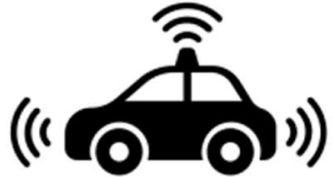

People with previous experience with AVs are more positive than people with no experience

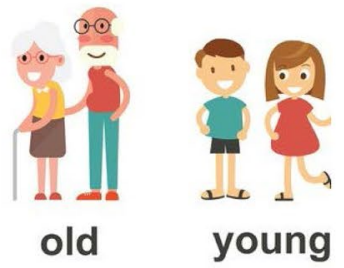

Younger people are more optimistic towards AVs than older people.

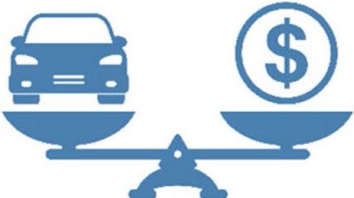

People in low GDP countries are more positive than people in medium and high GDP countries.

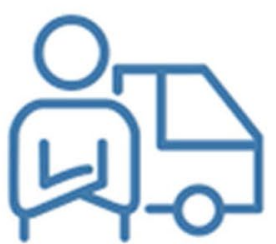

Although the risk on truck drivers due to AVs is real, but companies will always need drivers to perform some tasks.

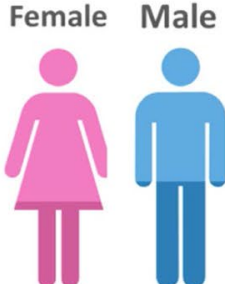

Males are more optimistic towards AVs than females.
People are not willing to pay more for AVs and only small proportion of people are willing to pay much more.

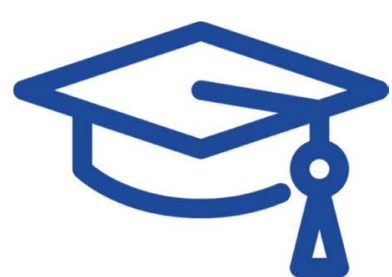

People with higher education levels are more positive towards AVs than people with lower education levels.

Fig. 9 The main results and conclusions of this study 
In other words, it is noteworthy that, although accessibility to the elderly and disabled was among the key benefits of $\mathrm{AVs}$, little to no research focused on the disabled to understand their acceptance of AVs. Additionally, results showed that old people are pessimistic about AVs, which contradicts the hypothesis that the elderly would benefit more from AVs.

- Cost of negative news (accidents): generally, people are highly concerned about AVs and the level of fear of AVs increase with the increase in the number of accidents reported. For example, the first AV fatal accident that involved Tesla's AV in 2016 had a significant impact on public acceptance of AVs and the level of interest in the AVs declined greatly after the accident that Tesla's share declined $1 \%$ in the day of the accident [48]. Although AVs might increase safety and reduce collisions, such news put the public against AVs. Consequently, AVs' providers must be aware of such an impact; especially in early stages when people are not familiar with the AV technology. Such accidents might harm the safety reputation of AVs and associate AVs with accidents in minds of the public which might discourage people from traveling in AVs.

- Liability issue: there is a critical issue in the liability between human drivers and manufacturers that will be raised in the near future. It seems non-logic to consider the occupant of the AV liable for an action in an AV, especially the main benefit of AVs is to allow the passengers to be involved in other activities. Thus, if the driver is asked to keep attention while the car is moving, then what is the difference between AVs and conventional vehicles? In such a case, will people accept to buy such a car? This point is critical for the future of AVs and requires additional studies to provide solutions that handle this liability issue.

Author contributions KO: literature search and review, research methodology, data preparation, data analysis, manuscript writing.

\section{References}

1. The Milwaukee Sentinel. Phantom Auto' will tour city. 8 December 1926

2. The Victoria Advocate. Power companies build for your new electric living. 24 Mars 1957

3. Davidson, P., Spinoulas, A.: Autonomous vehicles-what could this mean for the future of transport? AITPM 2015 National Conference. Sydney (2015)

4. Berrada, J., Leurent, F.: Modeling transportation systems involving autonomous vehicles: a state of the art. In: Transportation Research Procedia. 20th EURO Working Group on Transportation Meeting, EWGT 2017, Budapest, Hungary. 4-6 September 2017
5. Greenblatt, J.B., Shaheen, S.: Automated vehicles, on-demand mobility, and environmental impacts. Curr. Sustain. Renew. Energy Rep. 2, 74-81 (2015). https://doi.org/10.1007/s4051 8-015-0038-5

6. Mallozzi, P., Pelliccione, P., Knauss, A., Berger, C., Mohammadiha, N.: Autonomous Vehicles: state of the art, future trends, and challenges. In: Dajsuren, Y., van den Brand, M. (eds) Automotive Systems and Software Engineering. Springer, Berlin (2019). https://doi.org/10.1007/978-3-030-12157-0_16

7. Hartmans, A.: How Google's self-driving car project rose from a crazy idea to a top contender in the race toward a driverless future (2016). https://www.businessinsider.com/google-drive rless-car-history-photos-2016-10

8. O'Kane, S.: Uber debuts a new self-driving car with more failsafes (2019). https://www.theverge.com/2019/6/12/18662626/ uber-volvo-self-driving-car-safety-autonomous-factory-level

9. Staff, M.: Apple's vehicle project, focused on building an autonomous driving system (2019). https://www.macrumors.com/round up/apple-car/\#release_date

10. Taylo, T.: Top 8 self-driving startups to watch in 2018 and 2019 (2018). http://techgenix.com/self-driving-startups/

11. National Conference of State Legislatures. Autonomous vehicles/self-driving vehicles enacted legislation (2020). https ://www.ncsl.org/research/transportation/autonomous-vehiclesself-driving-vehicles-enacted-legislation.aspx

12. Laukkonen, J.: Are self-driving cars legal in your state? (2020). https://www.lifewire.com/are-self-driving-cars-legal-4587765

13. Othman, K.: Benefits of vehicle automation for public transportation operations. Curr. Trends Civ. Struct. Eng. (2020). https://doi. org/10.33552/CTCSE.2020.06.000646

14. Bansal, P., Kockelman, K.M., Singh, A.: Assessing public opinions of and interest in new vehicle technologies: an Austin perspective. Transp. Res. Part C 67, 1-14 (2016)

15. Othman, K.: Cities on the era of autonomous vehicles: a comparison between conventional vehicles and autonomous vehicles. 2020. In: The 3rd international conference on "Future of Smart Cities (FSC)" in collaboration with Xiamen University Malaysia, From 14th to 15th of November 2020

16. Antov, D., Banet, A., Barbier, C., Bellet, T., Bimpeh, Y., Boulanger, A., Brandstätter, C., Britschgi, V., Brosnan, M., Buttler, I., et al.: European Road Users' Risk Perception and Mobility: The Sartre 4 Survey. IFSTTAR, Paris (2012)

17. Othman, K.: Public transportation on the era of autonomous vehicles: exploring different scenarios. Civ. Eng. Res. J. 10(5), 555800 (2020). https://doi.org/10.19080/CERJ.2020.10.555800

18. Newcomb. "You Won't Need a Driver's License by $2040-\mathrm{CNN}$. com.” CNN. Cable News Network, 18 Sept. 2012. Web. 19 Nov. 2012. http://www.cnn.com/2012/09/18/tech/innovation/ieee2040-cars/index.html

19. Securing America's Future Energy (SAFE). America's workforce and the self-driving future: realizing productivity gains and spurring economic growth (2018)

20. KPMG. Connected and Autonomous Vehicles-The UK Economic Opportunity (2015).

21. Sivak, M., Schoettle, B.: Road safety with self-driving vehicles: general limitations and road sharing with conventional vehicles (University of Michigan, Ann Arbor, Transportation Research Institute, 2015-01) (2015).

22. Lee, C., Abdel-Aty, M.: Comprehensive analysis of vehiclepedestrian crashes at intersections in Florida. Accid. Anal. Prev. 37, 775-786 (2005)

23. Koopman, P., Wagner, M.: Autonomous vehicle safety: an interdisciplinary challenge. IEEE Intell. Transp. Syst. Mag. 9, 90-96 (2017). https://doi.org/10.1109/MITS.2016.2583491

24. Kalra, N.: Challenges and approaches to realizing autonomous vehicle safety. In: Presented to the Committee on Energy and 
Commerce Subcommittee on Digital Commerce and Consumer Protection United States House of Representatives. RAND Corporation, Santa Monica (2017)

25. Nishimoto, A.: All new tesla models will feature level 5-capable autopilot hardware (2016). https://www.motortrend.com/news/ new-tesla-models-will-feature-level-5-capable-autopilot-hardw are/

26. Walker, A.: Are self-driving cars safe for our cities? (2020). https ://www.curbed.com/2016/9/21/12991696/driverless-cars-safet y-pros-cons

27. Stewart, E.: Self-driving cars have to be safer than regular cars. The question is how much. vox, record (2019). https://www.vox. com/recode/2019/5/17/18564501/self-driving-car-morals-safet y-tesla-waymo

28. Tibken. Waymo CEO: autonomous cars won't ever be able to drive in all conditions. Cnet (2018). https://www.cnet.com/news/ alphabet-google-waymo-ceo-john-krafcik-autonomous-carswont-ever-be-able-to-drive-in-all-conditions/

29. University of Illinois College of Engineering. Platform for testing of autonomous vehicle safety. ScienceDaily. 2019. ScienceDaily, 25 October 2019.

30. www.sciencedaily.com/releases/2019/10/191025170813.htm

31. Martínez-Díaz, M., Soriguera, F.: Autonomous vehicles: theoretical and practical challenges. Transp. Res. Procedia 33, 275-282 (2018). https://doi.org/10.1016/j.trpro.2018.10.103

32. Casley, L., Jardim, A., Quartulli, A.: A Study of Public Acceptance of Autonomous Cars Interactive Qualifying Project. Worcester Polytechnic Institute, Worcester (2014)

33. Zmud, J., Sener, I., Wagner, J.: Consumer Acceptance and Travel Behavior Impacts of Automated Vehicles. Texas A\&M Transportation Institute, Texas (2016)

34. Rezaei, A., Caulfield, B.: Examining public acceptance of autonomous mobility. Trav. Behav. Soc. 21, 235-246 (2020). https:// doi.org/10.1016/j.tbs.2020.07.002

35. Schoettle, B., Sivak, M.: A survey of public opinion about autonomous and self-driving vehicles in the U.S., the U.K., and Australia (2014)

36. Schoettle, B., Sivak, M.: Motorists' preferences for different levels of vehicle automation (2015)

37. Kyriakidis, R., et al.: Public opinion on automated driving: Results of an international questionnaire among 5000 respondents. Transp. Res. Part F Traff. Psychol. Behav. 32, 127 (2015)

38. Greaves, S.P., Brett, S., Tony, A., Doina, O., Andrew T.C.: ITLSWP-18-18 autonomous vehicles down under: an empirical investigation of consumer sentiment (2018)

39. Obrecht, M., Knez, M., Sinko, S.: Analysis of public opinion on autonomous vehicles. In: 12th International Conference on Challenges of Europe: Innovative Responses for Resilient Growth and Competitiveness at: Bol (2017)

40. Jefferson, J., Mcdonald, A.: The autonomous vehicle social network: analyzing tweets after a recent tesla autopilot crash. In: The Human Factors and Ergonomics Society's 2019 International Annual Meeting at: Seattle, WA (2019)

41. Shearer, E., Gottfried, J.: News Use Across Social Media Platforms 2017|Pew Research Center. 2017. http://www.journalism .org/2017/09/07/news-use-across-social-mediaplatforms-2017/

42. Bohn, D. Elon Musk: negative media coverage of autonomous vehicles could be 'killing people' (2016). https://www.theverge. com/2016/10/19/13341306/elon-musk-negative-media-auton omous-vehicles-killing-people

43. Stewart, L., Musa, M., Croce, N.: Look no hands: self-driving vehicles' public trust problem. World economic Forum (2019). https://www.weforum.org/agenda/2019/08/self-driving-vehiclespublic-trust/

44. Eisenstein, P.: Not everyone is ready to ride as autonomous vehicles take to the road in ever-increasing numbers. CNBC
(2018). https://www.cnbc.com/2018/10/14/self-driving-carstake-to-the-road-but-not-everyone-is-ready-to-ride.html

45. Edmonds, E.: Three in four Americans remain afraid of fully self-driving vehicles (2019). https://newsroom.aaa. com/2019/03/americans-fear-self-driving-cars-survey/

46. Boudetten, N.: Autopilot cited in death of Chinese tesla driver. The New York Times (2016). https://www.nytim es.com/2016/09/15/business/fatal-tesla-crash-in-china-invol ved-autopilot-government-tv-says.html

47. Burke, K.: Lawsuit adds to scrutiny of Tesla's Autopilot. Automotive News (2016). https://www.autonews.com/artic le/20160919/OEM/309199962/lawsuit-adds-to-scrutiny-oftesla-s-autopilot.

48. McFarland, M.: Google's self-driving car takes some blame for a crash. The Sydney morning herald (2016). https://www.smh. com.au/technology/googles-selfdriving-car-takes-some-blame -for-a-crash-20160301-gn71z8.html

49. Levin, S., Woolf, N.: Tesla driver killed while using autopilot was watching Harry Potter, witness says. The Guardian (2016). https://www.theguardian.com/technology/2016/jul/01/ tesla-driver-killed-autopilot-self-driving-car-harry-potter

50. Guardian staff. Tesla car that crashed and killed driver was running on Autopilot, firm says. The Guardian (2018). https:// www.theguardian.com/technology/2018/mar/31/tesla-car-crash -autopilot-mountain-view

51. Levin, S.: Tesla fatal crash: 'autopilot' mode sped up car before driver killed, report finds. The Guardian (2018). https://www. theguardian.com/technology/2018/jun/07/tesla-fatal-crash-silic on-valley-autopilot-mode-report

52. Levin, S., Carrie, J.: Self-driving Uber kills Arizona woman in first fatal crash involving pedestrian. The Guardian (2018). https://www.theguardian.com/technology/2018/mar/19/uberself-driving-car-kills-woman-arizona-tempe

53. Tibken. Waymo CEO: Autonomous cars won't ever be able to drive in all conditions. Cnet. (2018). https://www.cnet.com/ news/alphabet-google-waymo-ceo-john-krafcik-autonomous -cars-wont-ever-be-able-to-drive-in-all-conditions/

54. BBC. Tesla hit parked police car 'while using Autopilot' (2018). https://www.bbc.com/news/technology-44300952

55. Murdock, J.: Tesla Model S Autopilot Sped Up Before Crashing Into Utah Fire truck. Newsweek (2018). https://www. newsweek.com/tesla-model-s-crash-car-autopilot-sped-justutah-firetruck-944251.

56. BBC. Tesla Model 3: Autopilot engaged during fatal crash (2019). https://www.bbc.com/news/technology-48308852

57. Coren, M.: Investigators found Autopilot was engaged in a Tesla crash in Florida. Quartz (2019). https://qz.com/1621235/ autopilot-was-engaged-in-a-2019-tesla-model-3-crash-in-flori da/

58. Coldewey, D.: Tesla explodes after crash on Russian highway. Tech Crunch (2019). https://techcrunch.com/2019/08/11/tesla -explodes-after-crash-on-russian-highway/

59. Boudette, M.: Autopilot Cited in Death of Chinese Tesla Driver. The New York Times (2016). https://www.nytim es.com/2016/09/15/business/fatal-tesla-crash-in-china-invol ved-autopilot-government-tv-says.html

60. Tynan, B., Yadron, D.: Tesla driver dies in first fatal crash while using autopilot mode. The Guardian (2016). https://www.thegu ardian.com/technology/2016/jun/30/tesla-autopilot-death-selfdriving-car-elon-musk

61. Cava, M.: Tesla crash in China renews spotlight on Autopilot. USA Today. 2016. https://www.usatoday.com/story/tech/ news/2016/09/14/tesla-crash-china-renews-spotlight-autop ilot/90367426/

62. Solomon, B.: Tesla autopilot enthusiast killed in first self-driving car death. Forbes. 2016. https://www.forbes.com/sites/brian 
solomon/2016/06/30/the-first-self-driving-car-death-launchestesla-investigation/?sh $=339716 \mathrm{~b} 17762$

63. McFarland, M.: For the first time, Google's self-driving car takes some blame for a crash. The Washington Post 2016. .https:// www.washingtonpost.com/news/innovations/wp/2016/02/29/ for-the-first-time-googles-self-driving-car-takes-some-blame -for-a-crash/

64. Davies, A.: Google's self-driving car caused its first crash. Wired. 2016. https://www.wired.com/2016/02/googles-self-driving-carmay-caused-first-crash/

65. Lee, D.: Google self-driving car hits a bus. BBC News. 2016. https://www.bbc.com/news/technology-35692845

66. Ziegler, C.: A Google self-driving car caused a crash for the first time. The Verge. 2016. https://www.theverge. com/2016/2/29/11134344/google-self-driving-car-crash-report

67. Levin, S., Woolf, N.: Tesla driver killed while using autopilot was watching Harry Potter, witness says the Guardian (2016). https:// www.theguardian.com/technology/2016/jul/01/tesla-driver-kille d-autopilot-self-driving-car-harry-potter

68. Bowerman, M.: Witness: Driver watched 'Harry Potter' as self-driving car crashed. USA Today (2016). https://www. usatoday.com/story/tech/nation-now/2016/07/01/navy-seal-vetkilled-using-teslas-autopilot-posted-close-call-video-month -ago/86592458/

69. Allen, N.: Tesla autopilot crash driver 'was watching Harry Potter movie', witness claims. The Telegraph (2016). https://www. telegraph.co.uk/news/2016/07/01/tesla-autopilot-crash-drive r-was-watching-harry-potter-movie-wit/

70. Associated Press. Tesla driver killed in autopilot crash 'was watching Harry Potter'. The New York Post (2017). https:// nypost.com/2016/07/02/tesla-driver-killed-in-autopilot-crash -was-watching-harry-potter/

71. Burke, K.: Tesla driver in fatal crash wasn't watching video, witness tells investigators. Automotive News (2017). https://www. autonews.com/article/20170619/MOBILITY/170619733/tesla -driver-in-fatal-crash-wasn-t-watching-video-witness-tells-inves tigators

72. Barnes, T.: Tesla autopilot caused car to accelerate before fatal crash, investigators find. The Independent (2018). https://www. independent.co.uk/news/world/americas/tesla-car-crash-autop ilot-acceleration-california-driver-death-investigation-lates t-a8389351.html

73. Heilweil, R.: Tesla needs to fix its deadly Autopilot problem. Vox (2020). https://www.vox.com/recode/2020/2/26/21154502/tesla -autopilot-fatal-crashes

74. The New York Times. Tesla autopilot system found probably at fault in 2018 crash (2020). https://www.nytimes.com/2020/02/25/ business/tesla-autopilot-ntsb.html

75. BBC News. Tesla Autopilot crash driver 'was playing video game' (2020). https://www.bbc.com/news/technology-51645566

76. Laris, M.: Fatal Tesla crash tied to technology and driver failures, NTSB says. The Washington Post (2020). https:// www.washingtonpost.com/local/trafficandcommuting/deadl y-tesla-crash-tied-to-technology-and-human-failures-ntsbsays/2020/02/25/86b710bc-574d-11ea-9b35-def5a027d470_ story.html

77. BBC News. Uber's self-driving operator charged over fatal crash (2020). https://www.bbc.com/news/technology-54175359

78. Hawkins, A.: Serious safety lapses led to Uber's fatal self-driving crash, new documents suggest. The Verge (2019). https://www. theverge.com/2019/11/6/20951385/uber-self-driving-crash-death -reason-ntsb-dcouments

79. Krisher, T.: Self-driving Uber car saw pedestrian but didn't brake, causing fatal accident: report. Global News (2018). https://globa lnews.ca/news/4229104/uber-self-driving-crash/
80. Cava, M.: Uber self-driving car kills Arizona pedestrian, realizing worst fears of the new tech. USA Today (2018). https://www. usatoday.com/story/tech/2018/03/19/uber-self-driving-car-kills -arizona-woman/438473002/

81. Wakabayashi, D.: Self-driving Uber car kills pedestrian in Arizona, where robots roam. The New York Times (2018). https:// www.nytimes.com/2018/03/19/technology/uber-driverless-fatal ity.html

82. McCausland, P.: Self-driving Uber car that hit and killed woman did not recognize that pedestrians jaywalk. NBC news. (2019). https://www.nbcnews.com/tech/tech-news/self-driving-uber-carhit-killed-woman-did-not-recognize-n1079281

83. Solon, O.: Tesla that crashed into police car was in 'autopilot' mode, California official says. The Guardian (2018). https:// www.theguardian.com/technology/2018/may/29/tesla-crash -autopilot-california-police-car

84. Allen, K.: Tesla on Autopilot crashes into parked police car. ABC news (2018). https://abcnews.go.com/US/tesla-autopilot-crash es-parked-police-car/story?id $=55525536$

85. Woodyard, C.: Tesla in Autopilot mode crashes into parked police cruiser. USA Today (2018). https://www.usatoday.com/ story/money/cars/2018/05/29/tesla-autopilot-self-driving-crash es-parked-police-car/654209002/

86. Gajanan, M.: Tesla in Autopilot Mode Crashes Into Parked Police Car in California. Fortune (2018). https://fortu ne.com/2018/05/29/tesla-autopilot-police-car-laguna-beach/

87. Feuer, F.: A Tesla on Autopilot rear-ended a parked police car, renewing safety concerns. CNBC (2019). https://www.cnbc. com/2019/12/09/tesla-on-autopilot-rear-ended-a-parked-polic e-car-on-the-highway.html

88. Hattem, J.: Tesla in autopilot mode accelerated seconds before crashing into firetruck: police. Global News (2018). https://globa lnews.ca/news/4231619/tesla-autopilot-firetruck-accident/

89. Associated Press. Tesla that crashed in Autopilot mode sped up before hitting truck-police. The Guardian (2018). https://www. theguardian.com/technology/2018/may/24/tesla-that-crashed-inautopilot-mode-sped-up-before-hitting-truck-police

90. CBS/AP. Police: Tesla in Autopilot sped up before Utah crash. CBS News (2018). https://www.cbsnews.com/news/police-tesla -in-autopilot-sped-up-before-utah-crash/

91. Sperling, J. Tesla in Autopilot Mode Accelerated Prior to Crash With Fire Truck, Police Say. Fortune (2018). https://fortu ne.com/2018/05/25/tesla-autopilot-crash-fire-truck/

92. The Washington Post. What self-driving cars can't recognize may be a matter of life and death (2018). https://www.washingtonpost. $\mathrm{com} /$

93. Associated Press. Utah driver sues Tesla after crashing in Autopilot mode. NBC News (2018). https://www.nbcnews.com/news/ us-news/utah-driver-sues-tesla-after-crashing-autopilot-moden906831

94. Chokshi, N.: Tesla autopilot system found probably at fault in 2018 crash. The New York Times (2020). https://www.nytim es.com/2020/02/25/business/tesla-autopilot-ntsb.html

95. Associated Press. NTSB: Tesla Autopilot was engaged before fatal Florida crash. Fox News (2019). https://www.foxnews.com/ auto/ntsb-tesla-autopilot-was-engaged-before-fatal-florida-crash

96. Brown, D.: Tesla stock drops after a report that Autopilot was engaged during a deadly crash in Florida. USA Today (2019). https://www.usatoday.com/story/tech/2019/05/17/teslas-autop ilot-active-during-fatal-model-3-crash-report-finds/3706981002/

97. Ohnsman, A.: Investigators say tesla model 3 driver killed in florida crash was using autopilot. Forbes (2019). https://www. forbes.com/sites/alanohnsman/2019/05/16/investigatorssay-tesla-model-3-driver-killed-in-florida-crash-used-autop ilot/?sh=3a6b1e8a77d5 
98. Cook, J.: Tesla's Autopilot was engaged before fatal Florida crash: NTSB. ABC News (2019). https://abcnews.go.com/ Politics/teslas-autopilot-engaged-fatal-florida-crash-ntsb/story ?id $=63107290$

99. Cuthbertson, A.: Self-driving Tesla 'kills' robot in Las Vegas crash, raising suspicions about Russian firm. The Independent (2019). https://www.independent.co.uk/life-style/gadgets-andtech/news/tesla-robot-crash-self-driving-car-promobot-putin -ces-2019-las-vegas-elon-musk-a8718866.html

100. ABC News. Tesla sued for 'defective autopilot' in the death of Florida driver. ABC News (2019). https://abcnews.go.com/ GMA/News/video/tesla-autopilot-mode-bursts-flames-crashesparked-truck-64920937

101. The Washington Post. A man died in a burning Tesla because its futuristic doors wouldn't open, lawsuit alleges (2019). https:// www.washingtonpost.com/

102. Daily News. Tesla explodes after crash on Russian highway. Daily News (2019). https://dailynewsgh.com/2019/08/12/tesla -explodes-after-crash-on-russian-highway/

103. Burke, K.: How does a self-driving car see? Camera, radar and lidar sensors give autonomous vehicles superhuman vision. Nvidia (2019). https://blogs.nvidia.com/blog/2019/04/15/howdoes-a-self-driving-car-see/

104. Viatech. Why sensor fusion is the key to self-driving cars. Viatech (2019). https://www.viatech.com/en/2019/10/why-senso r-fusion-is-the-key-to-self-driving-cars/

105. Kozma, P.: Why sensor fusion is critical for self-driving cars (2019). https://aimotive.com/blog/content/7321

106. Mitchell, H.B.: Multi-sensor data fusion: an introduction. Springer, Berlin (2007)

107. Hall, D.L., et al.: Handbook of multisensory data fusion. CRC Press, Boca Raton (2001)

108. Parhi, D.R., Singh, M.K.: Navigational strategies of mobile robots: a review. Int. J. Autom. Control 3(2/3), 114-134 (2009)

109. Mohamed, A., Ren, J., El-Gindy, M., Lang, H., Ouda, A.N.: Literature survey for autonomous vehicles: sensor fusion, computer vision, system identification and fault tolerance. Int. J. Autom. Control 12(4), 555-581 (2018)

110. Aitouche, A., Ould-Bouamama, B.: Sensor location with respect to fault tolerance properties. Int. J. Autom. Control 4(3), 298-316 (2010)

111. Brown, C., Durrant-Whyte, H., Leonard, J., Rao, B., Steer, B.: Distributed data fusion using Kalman filtering: a robotics application. In: Data Fusion in Robotics and Machine Intelligence, San Diego, CA, pp. 267-309 (1992)

112. Okello, N., Ristic, B.: Maximum likelihood registration for multiple dissimilar sensors. IEEE Trans. Aerosp. Electron. Syst. 39(3), 1074-1083 (2003)

113. Chen, S., Guo, Q., Leung, H., Bossé, E.: A maximum likelihood approach to joint image registration and fusion. IEEE Trans. Image Process. 20(5), 1363-1372 (2011)

114. Luo, R.C., Kay, M.G.: Data fusion and sensor integration: stateof-the-art 1990s. In: Abidi, M., Gonzalez, R. (eds.) Data Fusion in Robotics and Machine Intelligence, pp. 7-135. Academic Press, San Diego (1992)

115. Welch, G., Bishop, G.: Course 8: an Introduction to the Kalman filter', ACM SIGGRAPH (2001)

116. Caron, F., Duflos, E., Pomorski, D., Vanheeghe, P.: GPS/IMU data fusion using multisensor Kalman filtering: introduction of contextual aspects. Inf. Fusion 7(2), 221-230 (2006)

117. Li, X., Xu, Q., Chan, C., Li, B., Chen, W., Song, X.: A hybrid intelligent multi-sensor positioning methodology for reliable vehicle navigation. Math. Probl. Eng. 176947, 13 (2015)

118. Xian, Z., Hu, X., Lian, J.: Fusing stereo camera and low-cost inertial measurement unit for autonomous navigation in a tightlycoupled approach. J. Navig. 68(3), 434-452 (2015)
119. Ryu, J.H., Gankhuyag, G., Chong, K.T.: Navigation system heading and position accuracy improvement through GPS and INS data fusion. J. Sens.:1-6 (2016)

120. Crisan, D., Doucet, A.: A survey of convergence results on particle filtering methods for practitioners'. IEEE Trans. Signal Process. 50(3), 736-746 (2002)

121. Kurashiki, K., Fukao, T., Ishiyama, K., Kamiya, T., Murakami, N.: Orchard traveling UGV using particle filter based localization and inverse optimal control. In: 2010 IEEE/SICE International Symposium on System Integration, Sendai, pp. 31-36

122. Rigatos, G.G.: Extended Kalman and particle filtering for sensor fusion in motion control of mobile robots'. Math. Comput. Simul. 81(3), 590-607 (2010)

123. Rigatos, G.G.: Sensor fusion-based dynamic positioning of ships using extended Kalman and particle filtering. Robotica 31(3), 389-403 (2013)

124. Hiremath, S., van der Heijden, G., van Evert, F., Stein, A., terBraak, C.: Laser range finder model for autonomous navigation of a robot in a maize field using a particle filter. Comput. Electron. Agric. 100, 41-50 (2014)

125. Oniga, F., Nedevschi, S.: Processing dense stereo data using elevation maps: road surface, traffic isle, and obstacle detection. IEEE Trans. Veh. Technol. 59(3), 1172-1182 (2010)

126. Danescu, R., Nedevschi, S.: A particle-based solution for modeling and tracking dynamic digital elevation maps. IEEE Trans. Intell. Transp. Syst. 15(3), 1002-1015 (2014)

127. Min, K., Oh, J., Kim, B.: Traffic sign extract and recognition on unmanned vehicle using image processing based on support vector machine. 2011. In: 11th International Conference on Control, Automation and Systems, Gyeonggi-do, pp. 750-753

128. Schaub, A., Baumgartner, D., Burschka, D.: Reactive obstacle avoidance for highly maneuverable vehicles based on a two-stage optical flow clustering. IEEE Trans. Intell. Transp. Syst. 18(8), 2137-2152 (2016)

129. Realpe, M., Vintimilla, B.X., Vlacic, L.: A fault tolerant perception system for autonomous vehicles. In: 2016 35th Chinese Control Conference (CCC), Chengdu, pp. 6531-6536 (2016). https://doi.org/10.1109/ChiCC.2016.7554385

130. Dacier, M.: A fault forecasting approach for operational security monitoring. In: Cristian, F. (ed.) Dependable Computing for Critical Applications 4, vol. 9, pp. 215-217. Springer, Vienna (1995)

131. Isermann, R.: Fault-Diagnosis Applications: Model-Based Condition Monitoring: Actuators, Drives, Machinery, Plants, Sensors, and Fault-Tolerant Systems. Springer, Berlin (2011)

132. Meskin, N., Khorasani, K.: Fault detection and isolation: multivehicle unmanned systems. Springer, New York (2011)

133. Carlson, N.A.: Federated filter for fault-tolerant integrated navigation systems. In: Position Location and Navigation Symposium, 1988. Record. Navigation into the 21st Century. IEEE PLANS '88. IEEE, pp. 110-119 (1988)

134. Lawrence, Jr., P.J., Berarducci, M.P.: Comparison of federated and centralized Kalman filters with fault detection considerations. In: Position Location and Navigation Symposium, 1994. IEEE, pp. 703-710 (1994)

135. Aldridge, H.A.: Robot position sensor fault tolerance. Ph.D. 9713717, Carnegie Mellon University, United States-Pennsylvania (1996)

136. Zhang, Y., Jiang, J.: Bibliographical review on reconfigurable fault-tolerant control systems. Annu. Rev. Control 32(2), 229252 (2008)

137. Ducard, G.: Fault-Tolerant Flight Control and Guidance Systems: Practical Methods for Small Unmanned Aerial Vehicles, pp. 1-20. Springer, London (2009)

138. Benosman, M.: A survey of some recent results on nonlinear fault tolerant control. Math. Probl. Eng. 2010, 1-25 (2009) 
139. Fourlas, G., Karras, G.C., Kyriakopoulos, K.J.: Fault tolerant control for a 4-wheel skid steering mobile robot. In DX@ Safeprocess, pp. 177-184 (2015)

140. Vlantis, P., Bechlioulis, C.P., Karras, G., Fourlas, G., Kyriakopoulos, K.J.: Fault tolerant control for omni-directional mobile platforms with 4 mecanum wheels. In: IEEE International Conference on Robotics and Automation (ICRA), Stockholm, pp. 2395-2400 (2016)

141. Bader, K., Lussier, B., Schon, W.: A fault tolerant architecture for data fusion: a real application of Kalman filters for mobile robot localization. Robot. Autonom. Syst. 88, 11-23 (2017)

142. Lim, H.S.M., Taeihagh, A.: Algorithmic decision-making in avs: understanding ethical and technical concerns for smart cities. MDPI Sustain. 11, 5791 (2019)

143. Lin, P.: The ethics of autonomous cars. The Atlantic (2013)

144. https://www.theatlantic.com/technology/archive/2013/10/theethics-of-autonomous-cars/280360/

145. Worstall, T.: When should your driverless car from google be allowed to kill you? Forbes. 2014. https://www.forbes.com/ sites/timworstall/2014/06/18/when-should-your-driverless-carfrom-google-be-allowed-to-kill-you/\#36e3f970fa5b

146. Bonnefon, J., Shariff, A., Rahwan, L.: The social dilemma of autonomous vehicles. Science (2016). https://doi.org/10.1126/ science.aaf 2654

147. Lin, P.: Why ethics matters for autonomous cars. Autonomes Fahren: Technische, rechtliche und gesellschaftliche Aspekte, pp. 69-85. Springer, Berlin (2015)

148. Gerdes, J.C., Thornton, S.M.: Implementable ethics for autonomous vehicles. Autonomes Fahren: Technische, rechtliche und gesellschaftliche Aspekte. M. Maurer, J. C. (2015)

149. Goodall, N.J.: Ethical decision making during automated vehicle crashes. Transp. Res. Rec. J. Transp. Res. Board 2424(1), 58-65 (2014)

150. Gogoll, J., Müller, J.F.: Autonomous cars: in favor of a mandatory ethics setting. Sci. Eng. Ethics 23, 681-700 (2017). https ://doi.org/10.1007/s11948-016-9806-x

151. Luetge, C.: The German ethics code for automated and connected driving. Philos. Technol. 30, 547-558 (2017). https:// doi.org/10.1007/s13347-017-0284-0

152. http://moralmachine.mit.edu/

153. Awad, E., Dsouza, S., Kim, R., Schulz, J., Henrich, J., Shariff, A., Bonnefon, J., Rahwan, I.: The moral machine experiment. Nature 563(7729), 59-64 (2018)

154. Awad, E., Bonnefon, J., Shariff, A., Rahwan, I.: The thorny challenge of making moral machines: ethical dilemmas with self-driving cars. NIM Market Intell Rev 11(2), 42-47 (2019). https://doi.org/10.2478/nimmir-2019-0015

155. Awad, E., Dsouza, S., Kim, R., et al.: Reply to: Life and death decisions of autonomous vehicles. Nature 579, E3-E5 (2020). https://doi.org/10.1038/s41586-020-1988-3

156. Bonnefon, J.-F., Shariff, A., Rahwan, I.: Autonomous vehicles need experimental ethics: are we ready for utilitarian cars? 2015. https://pdfs.semanticscholar.org/13d4/56d4c53d7b03b90 ba59845a8f61b23b9f6e8.pdf

157. Bonnefon, J.-F., Shariff, A., Rahwan, I.: The social dilemma of autonomous vehicles. Science 352(6293), 1573-1576 (2016)

158. Hohenberger, C.: Do ethical defaults cost lives? The effect of defaults on people's choice for ethical principles in automated cars. In: Presented at the International Conference on Traffic and Transport Psychology. 2016. Brisbane, Australia, 2-5 August 2016

159. Frison, A.K., Wintersberger, P., Riener, A.: First person trolley problem: evaluation of drivers' ethical decisions in a driving simulator. In: Proceedings of the 8th International Conference on Automotive User Interfaces and Interactive Vehicular Applications Adjunct. ACM, New York, pp. 117-122 (2016)
160. Faulhaber, A., Dittmer, A., Blind, F., Wächter, M.A., Timm, S., Sütfeld, R., König, P.: Human decisions in moral dilemmas are largely described by Utilitarianism: virtual car driving study provides guidelines for ADVS. Retrieved September 8, 2017. https://arxiv.org/ftp/arxiv/papers/1706/1706.07332.pdf

161. Asimov, I. (ed.): Robot. Gnome Press, New York (1950)

162. Asimov, I.: Runaround. Astound. Sci. Fict. 29(1), 94-103 (1942)

163. Wang, H., Khajepour, A., Cao, D., Liu, T.: Ethical decision making in autonomous vehicles: challenges and research progress. In: IEEE Intelligent Transportation Systems Magazine (2020). https://doi.org/10.1109/MITS.2019.2953556.

164. Beavers, A.: 21 Moral machines and the threat of ethical nihilism. In: Robot Ethics: Ethical Social Implications Robotics, p. $333(2011)$

165. Driver, J.: The history of utilitarianism (2009)

166. Evans, L.: "Death in traffic: why are the ethical issues ignored?". Stud. Ethics Law Technol. (2008). https://doi. org/10.2202/1941-6008.1014

167. Russell, S., Norvig, P. (eds.): Artificial Intelligence: A Modern Approach. Prentice Hall, Upper Saddle River (2010)

168. Russell, S., Norvig, P. (eds.): Artificial Intelligence: A Modern Approach. Pearson Education, Malaysia (2016)

169. Arbesman, S.: "Explain it to me again, computer," Slate, Feb. 25, 2013. Accessed on: Feb. 25, 2013. [Online]. http:// www.slate.com/articles/technology/future_tense/2013/02/ will_computers_eventually_make_scientific_discoveries_we_ can_t_comprehend.single.html

170. Thornton, S., Pan, S., Erlien, S., Gerdes, J.: Incorporating ethical considerations into automated vehicle control. IEEE Trans. Intell. Transp. Syst. 18(6), 1429-1439 (2017). https:// doi.org/10.1109/TITS.2016.2609339

171. Lin, P. Abney, K., Bekey, G.: The ethical and social implications of robotics (2012)

172. Gerdes, J., Thornton, S.: Implementable ethics for autonomous vehicles. In: Fahren, A. (ed.) Ladenburg, pp. 87-102. Springer, Germany (2015)

173. Maurer, M., Gerdes, J., Lenz, B., Winner, H. (eds.): Autonomous Driving. Springer, Berlin (2016)

174. Radtke, R.: Role morality in the accounting profession: how do we compare to physicians and attorneys? J. Bus. Ethics 79(3), 279-297 (2008). https://doi.org/10.1007/s10551-007-9380-x

175. Wallach, W., Allen, C. (eds.): Moral Machines: Teaching Robots Right from Wrong. Oxford Univ, Press (2008)

176. Batavia, P., Pomerleau, D., Thorpe, C. (Eds.): Applying Advanced Learning Algorithms to ALVINN. The Robotics Institute, Carnegie Mellon Univ. (1996)

177. Stoeltje, G.: Policy brief: how does texas law change the legal landscape for automated vehicles. Texas A\&M Transportation institute. Transportation Policy Research Center (2017)

178. Bartolini, C., Tettamanti, T., Varga, I.: Critical features of autonomous road transport from the perspective of technological regulation and law. Transp. Res. Procedia 27, 791-798 (2017). https://doi.org/10.1016/j.trpro.2017.12.002

179. Taeihagh, A., Lim, H.: Governing autonomous vehicles: emerging responses for safety, liability, privacy, cybersecurity, and industry risks. Transp. Rev. 39(1), 103-128 (2019)

180. Slone, S.: State Laws on Autonomous Vehicles. The council of state governments (2016). http://knowledgecenter.csg.org/ $\mathrm{kc} /$ content/state-laws-autonomous-vehicles

181. Ilkova, V., Ilka, A.: Legal aspects of autonomous vehicles-an overview. In: 2017 21st International Conference on Process Control (PC), pp. 428-433 (2017)

182. James, M.A., Nidhi, K., Karlyn, D.S., Paul, S., Constantine, S., Oluwatobi, A.O.: Autonomous Vehicle Technology: A Guide for 
Policymakers. RAND Corporation. 2016. https://www.rand.org/ pubs/research_reports/RR443-2.html

183. Endsley, M.R.: Toward a theory of situation awareness in dynamic systems. Hum. Fact. 37(1), 32-64 (1995)

184. Petersen, L., Robert, L., Yang, X.J., Tilbury, D.: Situational Awareness, Driver's Trust in Automated Driving Systems and Secondary Task Performance. 2019. SAE International Journal of Connected and Autonomous Vehicles, Forthcoming10.2139/ ssrn. 3345543

185. Shinar, D.: Psychology on the road: the human factor in traffic safety. Wiley, New York (1978)

186. Treat, J.R., Tumbas, N.S., McDonal, S.T., Shinar, D., Hume, R.D., Mayer, R.E., Stansifer, R.L., Catellan, N.J.: Tri-level study of the causes of traffic accidents: Final report volume 1: Causal factor tabulations and assessments. Institute for research in public safety (DOT publication No. DOT HS-805 085). Indiana University, Bloomington (1997)

187. Gugerty, L.J.: Situation awareness during driving: explicit and implicit knowledge in dynamic spatial memory. J. Exp. Psychol. Appl. 3(1), 42-66 (1997)

188. Endsley, M.R.: Situation awareness in future autonomous vehicles: beware of the unexpected. In: Bagnara, S., Tartaglia, R., Albolino, S., Alexander, T., Fujita, Y. (eds) Proceedings of the 20th Congress of the International Ergonomics Association (IEA 2018). IEA 2018. Advances in Intelligent Systems and Computing, vol. 824. Springer, Cham (2019). https://doi.org/https://doi. org/10.1007/978-3-319-96071-5_32

189. Young, K.L., Regan, M.A., Lee, J.D.: Measuring the effects of driver distraction: Direct driving performance methods and measures. CRC Press, Boca Raton (2009). http://www.crenetbase.com/doi/abs/https://doi.org/10.1201/9781420007497.ch7

190. Hancock, P.A.: Automation: how much is too much? 2014. Ergonomics 57(3), 449-454 (2014). https://doi.org/10.1080/00140 139.2013.816375

191. Endsley, M., Kiris, E.: The out-of-the-loop performance problem and level of control in automation. Hum. Fact. J. Hum. Fact. Ergonom. Soc. (1995). https://doi.org/10.1518/0018720957 79064555

192. Miller, D., Sun, A., Ju, W.: Situation awareness with different levels of automation. In: 2014 IEEE International Conference on Systems, Man, and Cybernetics (SMC), San Diego, CA, pp. 688-693 (2014). https://doi.org/10.1109/SMC.2014.6973989

193. Sirkin, D., Martelaro, N., Johns, M., Ju, W.: Toward measurement of situation awareness in autonomous vehicles. In: Proceedings of the 2017 CHI Conference on Human Factors in Computing Systems (2017)

194. Tesla. Tesla Model S Owner Manual with Autosteer 7.0 (2015)

195. Tesla. Tesla Model S Owner Manual with Autosteer 7.1 (2016)

196. Lin, R., Ma, L., Zhang, W.: An interview study exploring Tesla drivers' behavioural adaptation. Appl. Ergonom. 72, 37-47 (2018). https://doi.org/10.1016/j.apergo.2018.04.006

197. Sun, X., Li, J., Tang, P., et al.: Exploring personalised autonomous vehicles to influence user trust. Cogn. Comput. 12, 11701186 (2020). https://doi.org/10.1007/s12559-020-09757-x

198. Richardson, N., et al.: Assessing Truck Drivers' and Fleet Managers' Opinions Towards Highly Automated Driving. Springer International Publishing, Cham (2015)

199. Piao, J., McDonald, M., Hounsell, N., Graindorge, M., Graindorge, T., Malhene, N.: Public views towards implementation of automated vehicles in urban areas. Transp. Res. Procedia $\mathbf{1 4}$ 2168-2177 (2016)

200. Cunningham, M., et al.: A survey of public opinion on automated vehicles in Australia and New Zealand. In: 28th ARRB International Conference. 2018, Brisbane, Queensland, Australia

201. Solon, O.: Team of hackers take remote control of Tesla Model S from 12 miles away. The Guardian (2016). https://www.thegu ardian.com/technology/2016/sep/20/tesla-model-s-chinesehack-remote-control-brakes

202. Feigenbaum, B.: Autonomous vehicles: a Guide for policy makers. Reason foundation (2018)

203. Wintersberger, P., et al.: Automated driving system, male, or female driver. In: Proceedings of the 8th International Conference on Automotive User Interfaces and Interactive Vehicular Applications-Automotive'UI 16, pp. 51-58 (2016)

204. Richardson, E., Davies, P.: The changing public's perception of self-driving cars (2018)

205. Bazilinskyy, P., et al.: An international crowdsourcing study into people's statements on fully automated driving. Procedia Manuf. 3, 2534-2542 (2015)

206. Gittleman, M., Monaco, K.: Truck-driving jobs: are they headed for rapid elimination? ILR Rev. 73(1), 3-24 (2020). https://doi. org/10.1177/0019793919858079

207. Abraham, H., et al.: Autonomous vehicles and alternatives to driving: trust, preferences, and effects of age. In: Transportation Research Board 96th Annual Meeting (2017).

208. Miller, S.A., Heard, B.R.: The environmental impact of autonomous vehicles depends on adoption patterns. Environ. Sci. Technol. 50(12), 6119-6121 (2016)

209. Compass Transportation and Technology prepared for: Securing America's Future Energy (SAFE). The Economic and Social Value of Autonomous Vehicles: Implications from Past NetworkScale Investments (2018)

210. Brown, A., Gonder, J., Repac, B.: An analysis of possible energy impacts of automated vehicle. (2014). https://doi. org/10.1007/978-3-319-05990-7_13

211. Clark, B.Y., Larco, N., Mann, R.F.: The impacts of autonomous vehicles and e-commerce on local government budgeting and finance (2017).

212. Tannert. Will you ever be able to afford a self-driving car? fastcompany (2014). https://www.fastcompany.com/3025722/willyou-ever-be-able-to-afford-a-self-driving-car

213. Nunes, Hernandez. The cost of self-driving cars will be the biggest barrier to their adoption. Harvard Business Review (2019). https://hbr.org/2019/01/the-cost-of-self-driving-cars-will-be-thebiggest-barrier-to-their-adoption

214. US department of transportation Autonomous vehicle fleet ownership and operating costs are expected to be half that of traditional vehicles by 2030-2040 (2017)

215. Ongel, et al.: Economic assessment of autonomous electric microtransit vehicles. MDPI Sustain. 11, 648 (2019)

216. Litman, T.: Autonomous vehicle implementation predictions, implications for transport planning. Victoria Transport Policy Institute (2020).

217. Moreno, A., Michalski, A., Llorca, C., Moeckel, R.: Shared Autonomous Vehicles Effect on Vehicle-Km Traveled and Average Trip Duration. J. Adv. Transp. (2018). https://doi. org/10.1155/2018/8969353

218. Munich (2020). https://civitas.eu/eccentric/munich

219. Sadik-Khan, J., Solomonow, S.: Fear of public transit got ahead of the evidence. The Atlantic (2020). https://www.theatlanti c.com/ideas/archive/2020/06/fear-transit-bad-cities/612979/

220. How coronavirus is disrupting public transit (2020). https://trans itapp.com/coronavirus

221. Harris, J.E.: The Subways Seeded the Massive Coronavirus Epidemic in New York City. National Bureau of Economic Research Working Paper No. 27021, Updated April 24, 2020. http://web. mit.edu/jeffrey/harris/HarrisJE_WP2_COVID19_NYC_24Apr-2020.pdf

222. Hallum, M.: Queens Councilman's call to shut down mass transit brushed aside by MTA, union (2020). https://www.amny.com/ transit/queens-councilmans-call-to-shut-down-mass-transitbrushed-aside-by-mta-union/ 
223. Spurr. As condition of COVID-19 bailout, Ontario asks TTC and others to consider private 'microtransit' in place of littleused bus routes, The star (2020). https://www.thestar.com/news/ gta/2020/08/17/as-condition-of-covid-19-bailout-ontario-asksttc-and-others-to-consider-private-microtransit-in-place-of-littl e-used-bus-routes.html

224. Burns, L., Jordan, W., Scarborough, B.: Transforming Personal Mobility. The Earth Institute, Columbia University (2012)

225. Crowe, S., Argo, A.I., Aurora, C., Pony, A.: Uber halt selfdriving tests due to COVID-19. The robot report (2020). https ://www.therobotreport.com/argo-ai-aurora-cruise-pony-ai-uberautonomous-vehicles-covid/

226. Tchir, J.: Could physical distancing reignite our excitement for autonomous driving? The globe and mail (2020). https://www. theglobeandmail.com/drive/mobility/article-could-social-dista ncing-reignite-our-excitement-for-autonomous-driving/

227. Grosbard, E.: Autonomous vehicles could be crucial in responding to future pandemics. The robot report (2020). https://www. therobotreport.com/autonomous-vehicles-vital-role-solving-futur e-pandemics/

228. Demaitre, E.: COVID-19 pandemic prompts more robot usage worldwide. The robot report (2020). https://www.therobotreport. com/covid-19-pandemic-prompts-more-robot-usage-worldwide/
229. Ford, T.: Autonomous shuttles help transport COVID-19 tests at Mayo Clinic in Florida. Mayo Clinic (2020). https://newsnetwor k.mayoclinic.org/discussion/autonomous-shuttles-help-transportcovid-19-tests-at-mayo-clinic-in-jacksonville/

230. Goscé, L., Johansson, A.: Analysing the link between public transport use and airborne transmission: mobility and contagion in the London underground. Environ. Health 17, 84 (2018). https ://doi.org/10.1186/s12940-018-0427-5

231. The Motional Consumer Mobility. The Motional Consumer Mobility Report. 2020. https://motional.com/mobilityreport/

232. Musulin, K.: Public AV enthusiasm growing amid pandemic: survey. Smart cities Dive (2020). https://www.smartcitiesdive .com/news/public-av-enthusiasm-growing-amid-pandemic-surve $\mathrm{y} / 585848 /$

233. Teale, C.: Poll: 3 in 4 Americans say AV tech 'not ready for primetime'. Smart cities Dive (2020). https://www.smartcitie sdive.com/news/poll-3-in-4-americans-say-av-tech-not-ready -for-primetime/578355/

234. Pani, A., Mishra, S., Golias, M., Figliozzi, M.: Evaluating public acceptance of autonomous delivery robots during COVID19 pandemic. Transp. Res. Part D Transp. Environ. 89, 102600 (2020). https://doi.org/10.1016/j.trd.2020.102600 